Purdue University Purdue e-Pubs

ECE Technical Reports

Electrical and Computer Engineering

7-3-2007

\title{
FSM-Based Model for Spatio-Temporal Event Recognition for HCS
}

\author{
Wamiq Manzoor Ahmed \\ Purdue University,wahmed@purdue.edu \\ Arif Ghafoor \\ Purdue University, ghafoor@purdue.edu \\ J. Paul Robinson \\ Purdue University
}

Follow this and additional works at: http://docs.lib.purdue.edu/ecetr

Ahmed, Wamiq Manzoor; Ghafoor, Arif; and Robinson, J. Paul, "FSM-Based Model for Spatio-Temporal Event Recognition for HCS" (2007). ECE Technical Reports. Paper 371.

http://docs.lib.purdue.edu/ecetr/371

This document has been made available through Purdue e-Pubs, a service of the Purdue University Libraries. Please contact epubs@purdue.edu for additional information. 


\title{
FSM-BASED MODEL FOR SPATIO-TEMPORAL EVENT RECOGNITION FOR HCS
}

\author{
Wamiq Manzoor Ahmed \\ Arif Ghafoor \\ J. Paul Robinson
}

TR-ECE-07-04

School of Electrical and Computer Engineering

1285 Electrical Engineering Building

Purdue University

West Lafayette, IN, 47907-1285 


\section{TABLE OF CONTENTS}

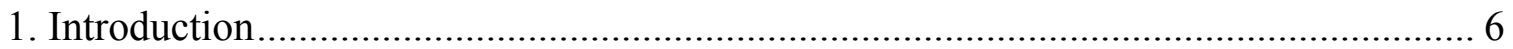

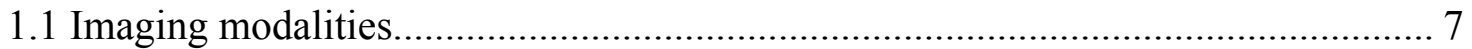

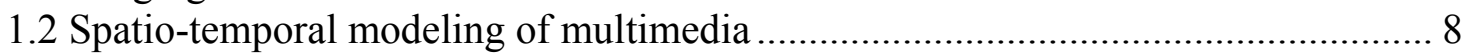

2. Multi-layered modeling of biological multimedia data ............................................. 8

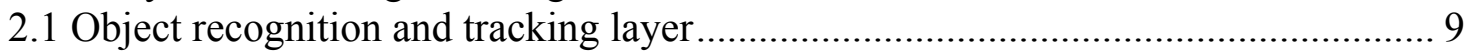

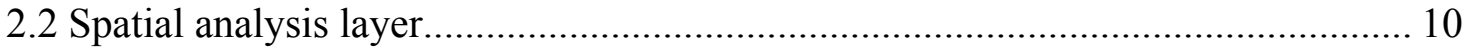

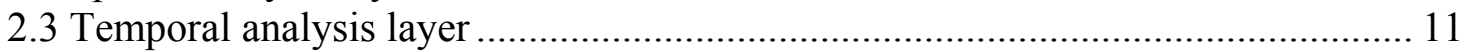

2.4 Knowledge extraction and representation layer................................................... 12

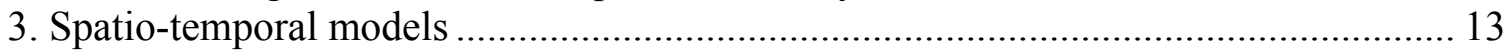

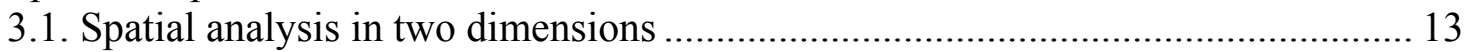

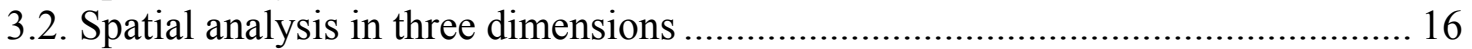

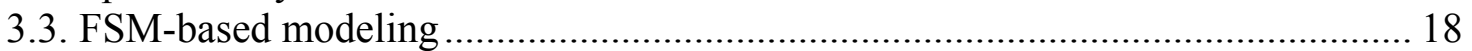

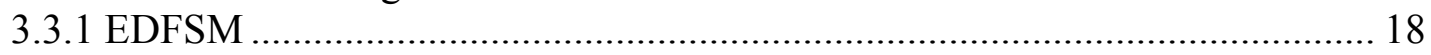

3.3.2 Event representation graph ..................................................................... 19

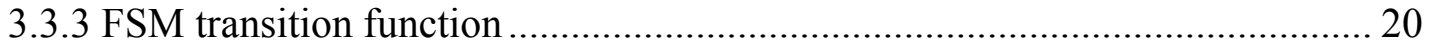

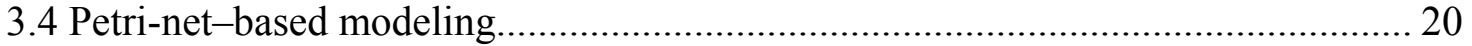

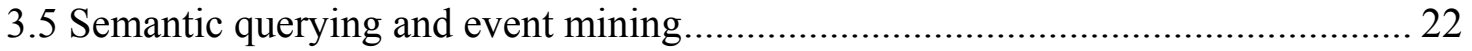

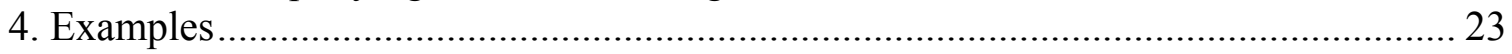

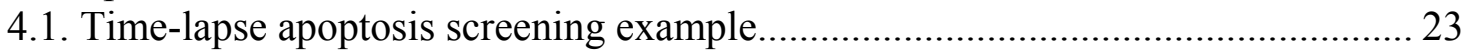

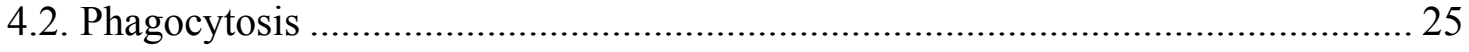

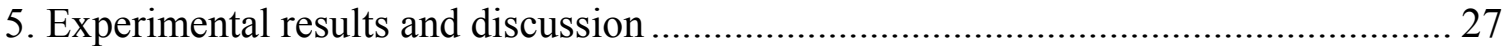

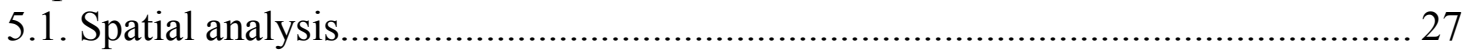

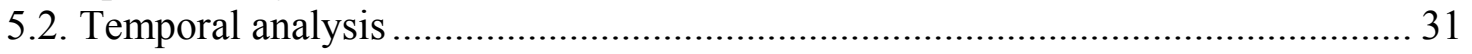

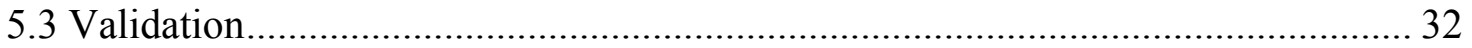

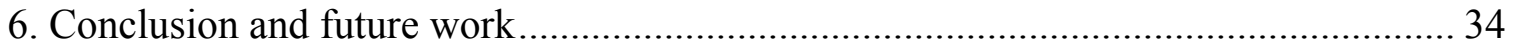

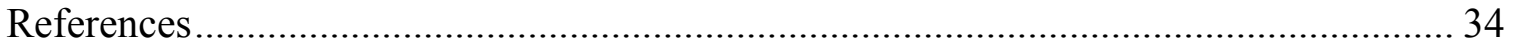




\section{LIST OF FIGURES}

Figure 1. Multi-layered abstraction

Figure 2. Different spatial representations. (a) Bounding box (b) Convex hull (c) Exact outline.

Figure 3. Projection of bounding boxes on coordinate axes for spatial analysis . 13

Figure 4. Hybrid algorithms for spatial analysis. ................................................... 16

Figure 5. Algorithms for 3D spatial analysis....................................................... 18

Figure 6. Symbols used for EDFSM and ERG. (a) start node (b) end node (c) intermediate node (d) transition. ................................................................ 19

Figure 7. FSM transition function.......................................................................... 20

Figure 8. (a) EDFSM for apoptosis event. S, V, E, and L correspond to start, live, early apoptotic, and late apoptotic states, (b) A composite event represented using petri-net, (c) A composite event with delay places represented using petri-net.

Figure 9. ERG representation of events in an imaging experiment ...................... 22

Figure 10. HL60 cells in different states during an apoptosis screen. (a) Hoechst 33342, (b) Annexin V FITC, (c) PI, (d) Merged image. The three images were pseudo-colored and merged to make the composite image. Blue, green and red colors correspond to Hoechst 33342, Annexin V FITC, and PI respectively. Cells in blue only are live, cells in blue and green are in early apoptotic state, and cells in blue, green, and red are in late apoptotic state. 24

Figure 11. Sequence of events constituting a phagocytosis event..... 25

Figure 12. EDFSM for a phagocytosis event, S1, S2, S3, S4, S5 correpond to start, disjoint, ingestion_start, ingestion_complete and phagocytosis_complete.

Figure 13. A representative set of images, (Upper left) Nuclei, (Lower left) Segmented nuclei, (Upper middle) Cytoplasm, (Lower middle) Segmented cytoplasm, (Upper right) Gold particles, (Lower right) Segmented gold particles.

Figure 14. (a) Convex hull of cytoplasm, (b) Convex hull of nuclei. Convex hull is a better spatial representation for nuclei than cytoplasm.

Figure 15. Particles localized inside cells (a) Histogram of the area of particles localized inside cytoplasm. (b) Histogram of the area of particles localized inside nucleus.

Figure 16. (a) Surface rendering of the 3D confocal data set simulating cells (b) Surface rendering of the 3D confocal data set simulating particles.

Figure 17. (a) Original population counts for time-lapse apoptosis study (b) Revised population counts for time-lapse apoptosis study. 


\section{LIST OF TABLES}

Table 1. Temporal relations

Table 2. Predicates for spatial analysis. B, M, S, O, C, E, CO refer to temporal relations as defined in Figure 4.

Table 3. Functions for maintaining ERG

Table 4. Cell states and corresponding spatial relations between Hoechst $(H)$, Annexin-V-FITC(A), and $\mathrm{PI}(\mathrm{P})$.

Table 5. State transition table corresponding to the EDFSM of Figure 5. Actions $\mathrm{cr}$ and inc correspond to the create and increment functions that create a node in ERG or increment the duration of a node in ERG. Spatial relations refer to the ones defined in Table 2

Table 6. State transition table corresponding to the EDFSM of Figure 10. Actions create, inc and rb create a node in ERG, increment duration, and rollback to an appropriate state respectively.

Table 7. Comparison of accuracy of different algorithms. EO algorithm is used as the base case

Table 8. Comparison of speed of different algorithms.

Table 9. Comparison of the runtime for different 3D-spatial-analysis algorithms. An image set containing 50 z-slices was used. Each slice was $500 \times 500$ pixels.

Table 10. Performance of artifact removal rule on synthetic data. 


\title{
FSM-BASED MODEL FOR SPATIO-TEMPORAL EVENT RECOGNITION FOR HCS
}

\author{
Wamiq Manzoor Ahmed ${ }^{1,3}$, Arif Ghafoor ${ }^{1}$, J. Paul Robinson ${ }^{2,3}$ \\ ${ }^{1}$ School of Electrical and Computer Engineering, 465 Northwestern Avenue, \\ ${ }^{2}$ Weldon School of Biomedical Engineering, 206 South Intramural drive \\ ${ }^{3}$ Purdue University Cytometry Laboratories, 1203 West State Street, \\ West Lafayette, IN 47907
}

\begin{abstract}
High-throughput biological imaging promises to be a powerful tool for modern biological research. These imaging technologies are used to monitor the behavior of large populations of cells in response to different experimental conditions. The resulting knowledge can help in drug discovery and in understanding biological phenomena. Different imaging modalities are used for high-throughput imaging. These include 2D fluorescence images, 3D confocal datasets, time-lapse image sequences, and multispectral images. Integrated analysis of these multi-modality spatial, temporal, and spectral data sets for extracting quantitative knowledge is challenging and requires new modeling and data processing tools. This paper presents a multi-layered architecture and spatio-temporal models for analysis of such data. The analysis is divided into low-level and high-level processing. At the lower level, issues like segmentation, tracking and object recognition are addressed, and at the high level, finite state machine- and Petrinet-based models are used for spatio-temporal event recognition. This approach provides a mechanism for extracting high-level spatio-temporal knowledge, and improves searching and retrieval of high-throughput biological imaging data by means of semantic and conceptual queries.
\end{abstract}




\section{Introduction}

Recent advances in high-speed cameras, automation technologies, fluorescent probes, and optics have given rise to the field of high-content/high-throughput screening (HCS/HTS) [1]. HCS systems are used for monitoring the behavior of large populations of cells under different experimental conditions. The resulting knowledge can help in drug target selection, in understanding disease mechanisms, and in deciphering complex biological processes [2]. The wealth of data provided by these sophisticated technologies combined with the genomic and proteomic information can help unravel complex biological processes. The realization of this dream requires intelligent data processing tools that can extract objective and quantitative information from large volumes of HCS data sets [3]. Traditional visual examination is extremely slow and prone to human error. There is a need for intelligent multimedia interpretation tools that can extract high-level knowledge from large multi-modality biological imaging data sets. In this paper we describe a multi-layered architecture, and models for extracting spatio-temporal information from biological imaging datasets.

Traditionally, biologists have used small-scale experimentation followed by visual examination for understanding biological processes. Based on the limited experimental information, hypotheses are developed which are then tested by further experimentation. This approach, though satisfactory for small-scale biology, has serious shortcomings for high-throughput technologies. A tremendous amount of genomic and proteomic data has been made available in recent years by successful collaborative efforts [4]. It is clear that understanding of highly complex biological processes requires the integrated analysis of 
cells, tissues, and higher-level organisms instead of just one component in isolation. Our work focuses on developing data-modeling schemes and automated knowledge extraction tools for optical imaging of biological specimens using different imaging modalities.

\subsection{Imaging modalities}

High-throughput imaging devices combine sophisticated optics and cameras with automation technologies and provide large volume of imaging data for cell-based systems. Different imaging modalities can be used for this purpose [5]. Bright-field, dark-

field, phase-contrast, and multi-photon imaging generate 2D images. As most biological specimens are three dimensional, this 2D information is not sufficient. Confocal microscopy provides 3D image sets that can be used for 3D reconstruction and visualization. Confocal microscopes use a pinhole in the optical path that rejects most of the out-of-focus light. This way the image is produced only from a thin section of the sample. By collecting many such sections, a 3D representation of the sample is generated. Multispectral imaging is used for differentiating fluorochromes with similar spectra or for separating autofluorescence from fluorochrome fluorescence [6]. This imaging modality uses various types of filters to collect sample information at different spectral bands. Moreover, dynamic biological processes require time-lapse imaging, and many biological applications require combinations of these imaging modalities. This gives rise to 5dimensional image sets (three spatial, one temporal, and one spectral dimension). Integrated analysis of these multi-modality spatial, temporal, and spectral data sets for extracting quantitative knowledge is challenging and requires new modeling and data processing tools. 


\subsection{Spatio-temporal modeling of multimedia}

Numerous multimedia modeling techniques have been developed in the past [7-9]. Applications of these modeling schemes range from automatic surveillance to summarization and indexing of sports and news videos. However, biological multimedia data have only recently started attracting attention. Objective and quantitative analysis of biological data sets requires robust modeling approaches and intelligent analysis tools. There is a pressing need for biologists and multimedia experts to work together and jointly solve the complicated problems of biological multimedia analysis. Many applications can benefit from automatic semantic analysis of biological data. Analysis and quantitation of cell phenotypes help in understanding the response of cells to different environmental conditions. This information is also useful for understanding the function of cells and the alterations in their function due to diseases. Different cellular phenomena like apoptosis, phagocytosis, cell division, motility, etc. can be studied in a quantitative fashion, and effects of different drugs or experimental conditions can be ascertained. These techniques can provide a wealth of information for systems biology approaches and can potentially speed up the drug discovery process.

\section{Multi-layered modeling of biological multimedia data}

Knowledge extraction from biological multimedia data is extremely challenging and requires modeling at multiple levels. At the lower level, it requires sophisticated image processing techniques for preprocessing, segmentation, pattern recognition, and cell tracking, and at the higher level it necessitates the modeling of spatial and temporal dynamics of biological systems to extract high-level semantics. A multi-layered modeling 
scheme as shown in Figure 1 is therefore necessary to capture not only the low-level details of biological objects but also the higher-level semantics. Biological applications vary in terms of their semantic analysis requirements. Some applications only require quantification of simple parameters like size or fluorescence intensity whereas others require recognition of biological objects or quantitative analysis of the evolution of biological objects over time. A multi-level modeling approach therefore meets the requirements of a variety of biological applications. This paper focuses on spatial and temporal analysis which constitutes the two middle layers shown in Figure 1.

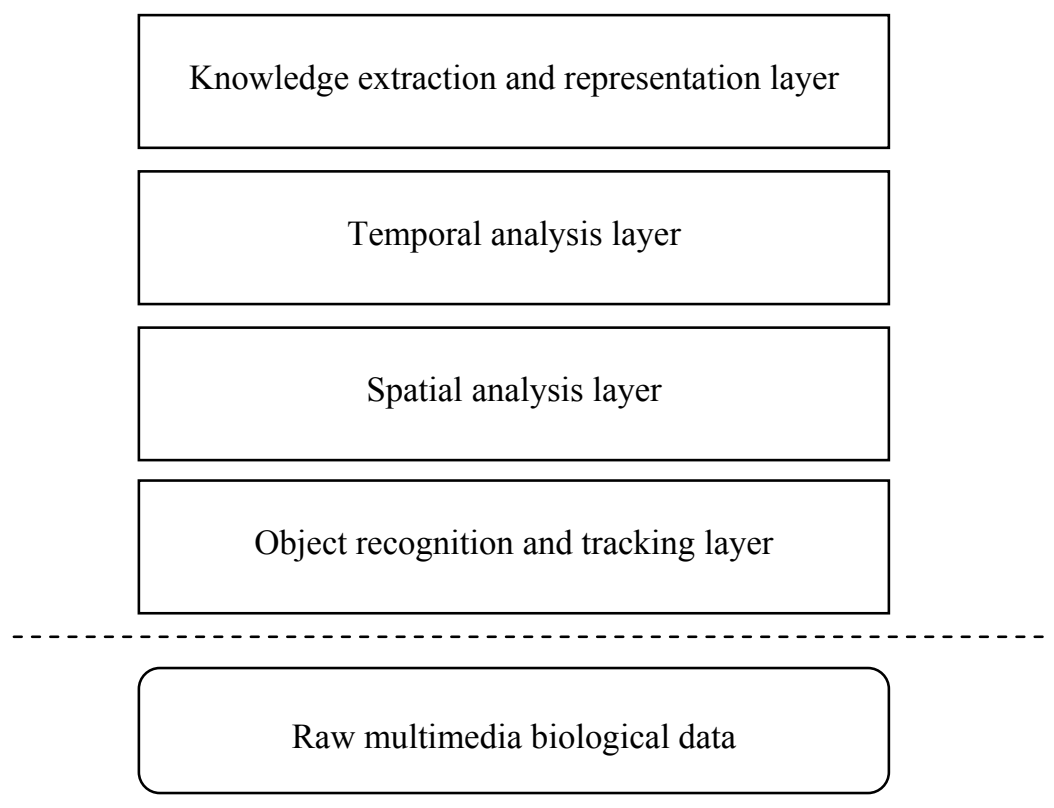

Figure 1. Multi-layered abstraction

\subsection{Object recognition and tracking layer}

Raw multimedia biological data may consist of 2D images, 3D confocal data sets, multispectral images, and time-lapse microscopic videos. Analysis of acquired images starts at the object recognition and tracking layer. The first step is to separate objects of interest from background. This step is made more challenging by variations in biological 
objects and in acquisition parameters of imaging instruments, such as illumination conditions $[10,11]$. Some preprocessing steps like illumination normalization, denoising and deconvolution are generally needed before segmentation [12]. Sample preparation may also improve segmentation accuracy. Dilute samples in which cells are not clustered may be more suitable for automated segmentation. Cell segmentation algorithms aim at separating objects of interest from the background. Algorithms based on deformable templates and watershed transform have been successfully used for this purpose [13-15]. Some algorithms first segment the nuclei and then use them for segmenting the cytoplasm in fluorescence imaging [16].

Object recognition is the next step after segmentation. Object features like size, shape, and texture are first extracted, and then classification algorithms like neural networks, decision trees, or support vector machines are used [17]. Object recognition requires prior training of these algorithms with examples of objects of interest. This layer also provides functionality for tracking cell movements. Tracking of cellular and molecular objects is a challenging problem that has attracted significant attention [18-20]. Cell tracking algorithms based on correlation, deformable models, image level sets and mean shift have been proposed in the literature [18-20]. Appropriate algorithms can be used for tracking objects and all per-object information can be stored using appropriate data structures.

\subsection{Spatial analysis layer}

Once different objects in a frame are identified, this information is passed onto the spatial analysis layer. Study of spatial distribution of cells and spatial relationships among 
biological objects is essential for understanding biological processes. Spatial distribution analysis tries to find patterns in the distribution of cells. Such patterns are useful for studying the behavior of cells and changes in these patterns may indicate disease states. Spatial relationship analysis tries to localize objects like drug molecules or particles like quantum dots with respect to intracellular objects. As biological objects are mostly transparent, contrast agents called markers are used to stain specific cell sites. These markers fluoresce at their emission bands when illuminated with proper excitation. When multiple markers are used, spatial relations between different cell sites are of interest. For example, to check if a drug stains a particular cell site, the drug and cell sites of interest can be stained with different dyes and then spatial relations between these fluorescent objects can be analyzed to localize the drug inside cells. Formalisms for specifying spatial relations among different objects are presented in section 3 .

\subsection{Temporal analysis layer}

Many biological processes are dynamic in nature and happen over a period of time. Cells undergoing apoptois or mitosis are examples of temporal events. Different environmental conditions can affect the timing of these events. A study of the temporal dynamics of such processes yields valuable information. The temporal analysis layer extracts this information. We introduce FSM and Petri-net-based formalisms in section 3 for specifying temporal events. The FSM approach models cells in terms of their features, and spatio-temporal events in terms of changes in these features with the passage of time. The Petri-net approach is used to model temporal constraints of complex events. Templates of spatio-temporal events are provided by user, and instances of those events in the imaging data are identified at this layer. 


\subsection{Knowledge extraction and representation layer}

The final step of processing is the representation of extracted knowledge. The knowledge extraction and representation layer deals with this issue. Spatial and temporal events that are identified by lower layers are analyzed to identify patterns in the data. This conceptual information along with spatial and temporal information is then stored. In other work, we have proposed an XML-based language for storing this high-level semantic information [21]. This representation serves three purposes. Firstly, it makes it possible to store the semantic information in a machine-readable format so that this information can be shared with other research groups. Secondly, it makes it possible to use data mining tools for identifying hidden patterns in multimedia imaging data. Thirdly, the same semantic information serves as high-level metadata and makes semantic querying and retrieval possible.

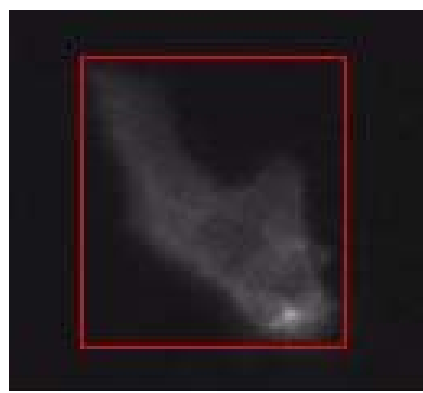

(a)

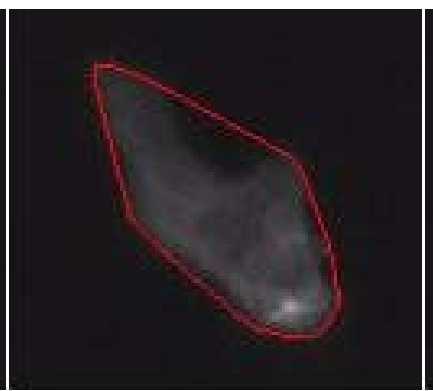

(b)

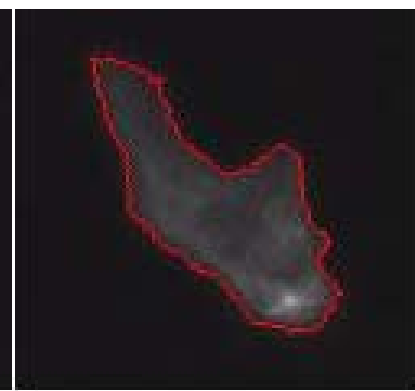

(c)

Figure 2. Different spatial representations. (a) Bounding box (b) Convex hull (c) Exact outline

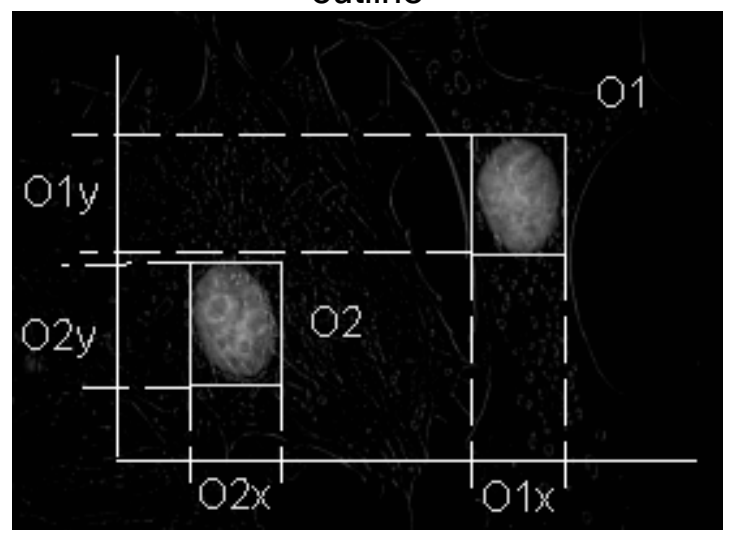


Figure 3. Projection of bounding boxes on coordinate axes for spatial analysis

\section{Spatio-temporal models}

In our data model, cells and other biological entities are modeled as objects with specific attributes like color, size, shape, etc., and events are modeled in terms of specific values of attributes of participating objects along with spatial relationships between these objects.

\subsection{Spatial analysis in two dimensions}

Inter-object spatial relations describe the relationships between the locations of two objects in two or three dimensional space. Orthogonal (north, south) and containment relations (contained, disjoint) are the two types of spatial relations defined for objects in images [9]. The first step for establishing spatial relations is to have a spatial representation of objects. Figure 2 shows different spatial representations for objects that include bounding box (BB), convex hull $(\mathrm{CH})$ and exact outline $(\mathrm{EO})$. A bounding box around the object is a simple spatial representation. Even though it is imprecise compared to other approaches, its simplicity and speed make it an attractive choice for situations where a high level of accuracy is not required, for example samples containing lowdensity, non-touching cells. For the bounding box case, inter-object spatial analysis can be carried out using projections on coordinate axes as shown in Figure 3. An extension of temporal relations shown in Table 1 [22] to two and three dimensions is then used to describe the inter-object relationships, as described in [23]. As shown in Table 1, there are seven basic relations between temporal intervals, six of which have inverses whereas 'equals' relation has no inverse. This gives a total of 13 relations between temporal intervals. An extension of this approach to two and three dimensions yields $13 \times 13$ relations in two dimensions and $13 \times 13 \times 13$ relations in three dimensions [24]. Other 
spatial representations include convex hull of the object and the exact outline which are more accurate compared to the bounding box approach. EO is the most accurate of the three spatial representations. For $\mathrm{CH}$ and $\mathrm{EO}$ representations, polygon intersection tests are used to determine spatial relations between objects. We define predicates for these three cases using different spatial representations in Table 2.

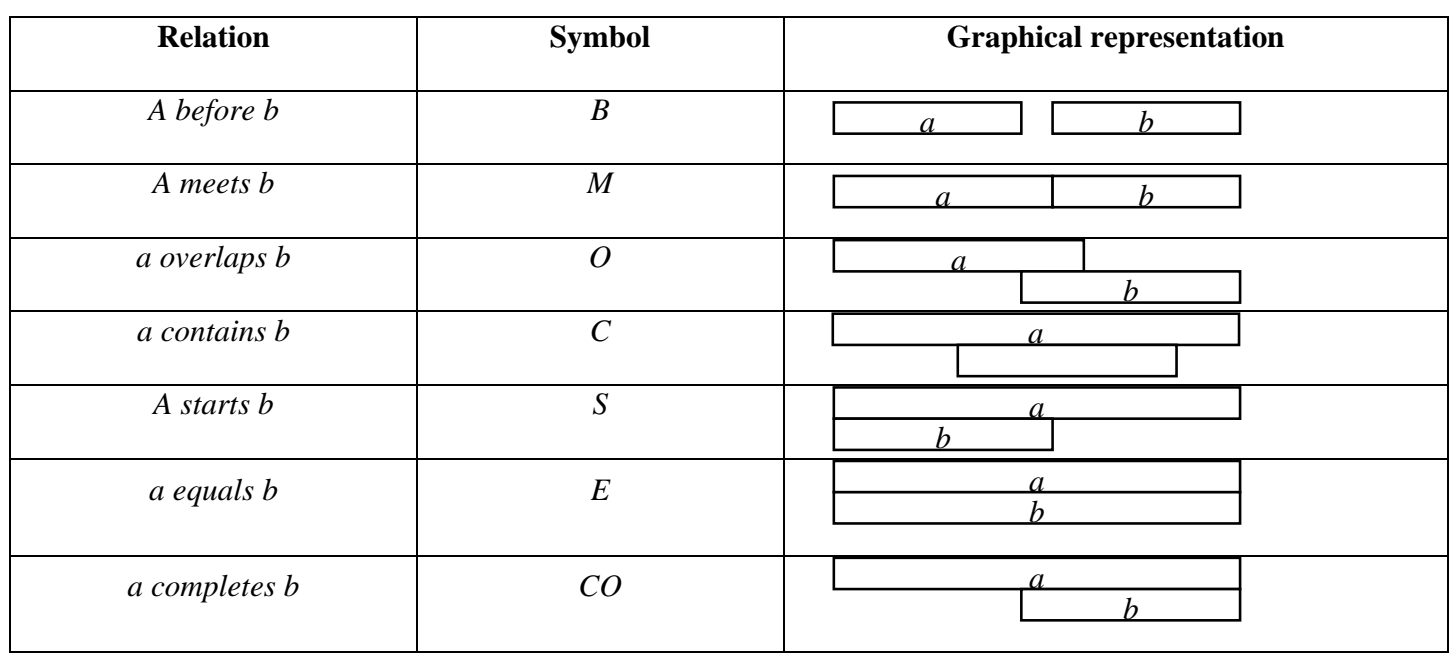

\section{Table 1. Temporal relations}

We now discuss the computational complexity of carrying out spatial analysis using different spatial representations. The bounding-box approach uses a fixed number of point comparisons for establishing spatial relations, hence it has complexity $\mathrm{O}(1)$. The polygon intersection test for convex polygons with $\mathrm{m}$ and $\mathrm{n}$ vertices has complexity $\mathrm{O}(\mathrm{m}+\mathrm{n})$, whereas the polygon intersection test for two arbitrary polygons with $\mathrm{n}$ and $\mathrm{m}$ vertices can have complexity $\Omega(\mathrm{nm})$ [25]. Hence, spatial analysis using the bounding box is the fastest, but is inaccurate compared with other techniques, whereas spatial analysis using exact outline is the most accurate but the slowest at the same time. Spatial analysis using convex hull falls between bounding box and exact outline in terms of speed and accuracy. A combination of these approaches can be used to provide trade-off between speed and accuracy. A crude analysis can first be performed using BB, followed by a 
more detailed analysis using $\mathrm{CH}$ or $\mathrm{EO}$ or a combination of both. In Figure 4 we present three different algorithms that combine $\mathrm{BB}, \mathrm{CH}$ and $\mathrm{EO}$ based analysis. These algorithms make use of the fact that the exact outline of an object is fully contained in the convex hull, and the convex hull is fully contained in the bounding box. Inputs to these algorithms are lists of objects between which spatial relations are to be established, for example, a list of nano particles and a list of cells.

\begin{tabular}{|c|c|}
\hline Predicate & Explanation \\
\hline $\operatorname{projx}(a)$ & Returns the projection on the $\mathrm{x}$ axis of the bounding box of object $a$. \\
\hline $\operatorname{projy}(a)$ & Returns the projection on the $y$ axis of the bounding box of object $a$. \\
\hline $\operatorname{projz}(a)$ & Returns the projection on the $\mathrm{z}$ axis of the bounding box of object $a$. \\
\hline $\begin{array}{l}\text { BOverlap } \\
(a, b)\end{array}$ & $\begin{array}{l}\text { Returns true if the following condition holds } \\
{\left[\left(\begin{array}{l}O(\operatorname{projx}(a), \operatorname{projx}(b)) \wedge \\
(M . O . C . S . C O . E(\operatorname{projy}(a), \operatorname{projy}(b)))\end{array}\right) \vee\left(\begin{array}{l}O(\operatorname{projy}(b), \operatorname{projy}(a)) \wedge \\
(M . O . C . S . C O . E(\operatorname{projx}(a), \operatorname{projx}(b)))\end{array}\right)\right.}\end{array}$ \\
\hline BDisjoint $(a, b)$ & $\begin{array}{l}\text { Returns true if the following condition holds } \\
{\left[\begin{array}{l}B(\operatorname{proj} x(a), \operatorname{projx}(b)) \vee B(\operatorname{projy}(a), \operatorname{projy}(b)) \vee \\
B(\operatorname{proj} x(a), \operatorname{proj} x(b)) \vee B(\operatorname{projy}(a), \operatorname{projy}(b))\end{array}\right]}\end{array}$ \\
\hline $\begin{array}{l}\text { BEnclosed } \\
(a, b)\end{array}$ & $\begin{array}{l}\text { Returns true if the following condition holds } \\
{\left[\text { C.S.CO }(\operatorname{projx}(a), \operatorname{projx}(b))^{\wedge} C . S . C O(\operatorname{projy}(a), \operatorname{projy}(b))\right]}\end{array}$ \\
\hline $\begin{array}{l}\text { CContain } \\
(a, b)\end{array}$ & Returns true if the convex hull of a is contained within the convex hull of $b$. \\
\hline $\begin{array}{l}\text { COverlap } \\
(a, b)\end{array}$ & Returns true if the convex hull of a has an overlap with the convex hull of $b$. \\
\hline $\begin{array}{l}\text { CDisjoint } \\
(a, b)\end{array}$ & Returns true if the convex hull of a is disjoint from the convex hull of $b$. \\
\hline $\begin{array}{l}\text { PContain } \\
(a, b)\end{array}$ & Returns true if the exact outline of $a$ is contained within the exact outline of $b$. \\
\hline $\begin{array}{l}\text { POverlap } \\
(a, b)\end{array}$ & Returns true if the exact outline of a has an overlap with the exact outline of $b$. \\
\hline $\begin{array}{l}\text { PDisjoint } \\
(a, b)\end{array}$ & Returns true if the exact outline of a is disjoint from the exact outline of $b$. \\
\hline Bound(a) & Returns the corner pixels of the bounding box of object $a$. \\
\hline PList(a) & Returns the list of pixels of the object $a$. \\
\hline $\operatorname{Rel}(a, b)$ & Returns the spatial relation between objects $a$ and $b$. \\
\hline PeriphObjs $(a, B)$ & $\begin{array}{l}\text { Returns all objects in the object list } B \text { that lie on the periphery of the object } a \text {. } \\
\forall b \in B \mid \operatorname{Rel}(a, b) \neq \operatorname{Rel}(\operatorname{Erosion}(a), b)\end{array}$ \\
\hline
\end{tabular}

Table 2. Predicates for spatial analysis. B, M, S, O, C, E, CO refer to temporal relations as defined in Figure 4. 


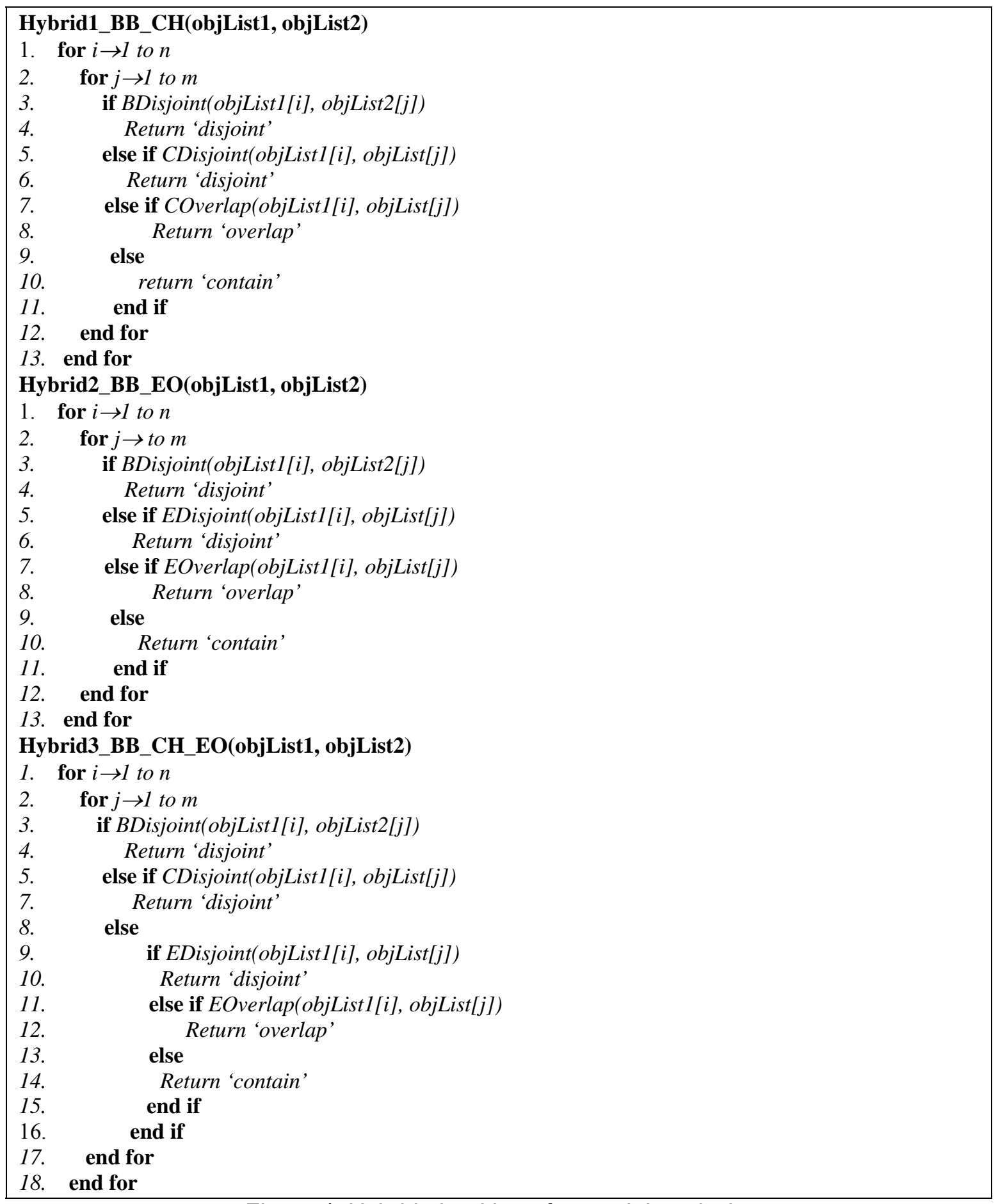

Figure 4. Hybrid algorithms for spatial analysis.

\subsection{Spatial analysis in three dimensions}


For 3D spatial analysis, we extend the bounding box approach to three dimensions. The predicates defined in Table 2 can be extended to three dimensions by using the projection of the bounding box on the $\mathrm{z}$ axis. We present three different algorithms for 3D spatial analysis. The first algorithm (3DBB) is based on the extension of two-dimensional bounding-box analysis to 3D. The other two algorithms (3DHybrid and 3DPixComp) are presented in Figure 5. 3DHybrid uses the bounding box representation for the smaller objects (particles), and extracts the eight corners of the bounding box for spatial analysis. If all the corners points of the bounding box are found to be inside the three dimensional representation of the cell, then the whole particle is localized inside the cell. If all corner of the bounding box are located outside, then the particle is localized outside the cell and otherwise it has a partial overlap. The third algorithm (3DPixelComp) uses individual pixels of particles to check if they are contained within the cells.

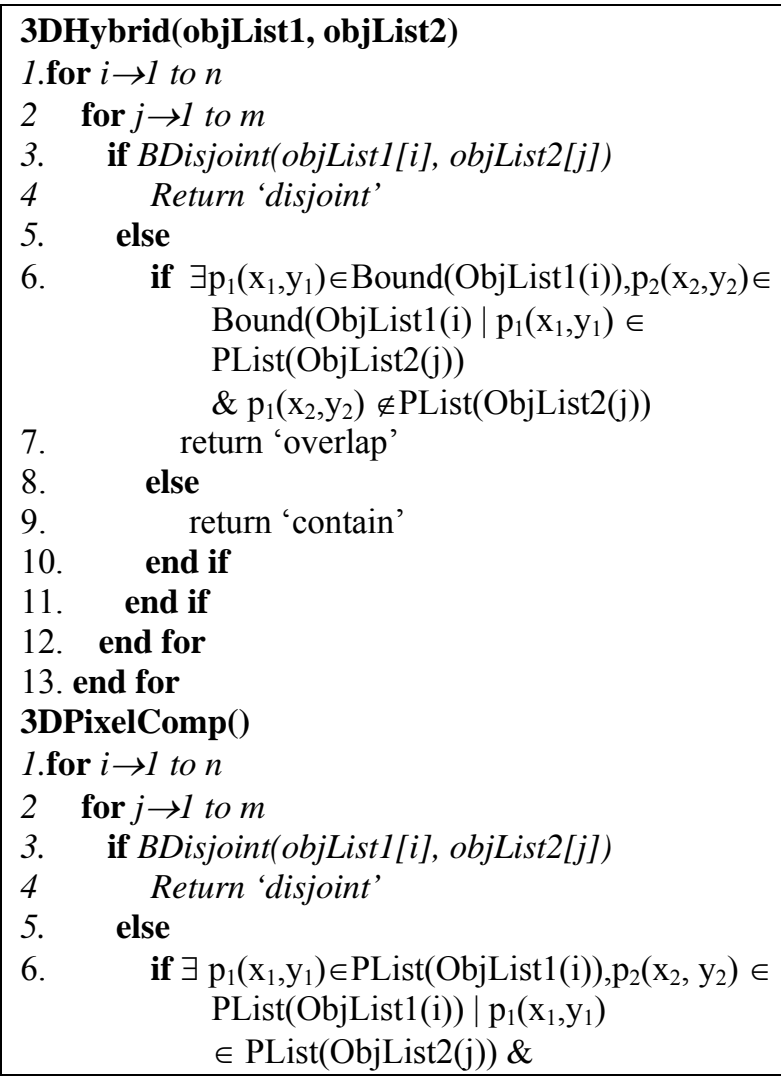




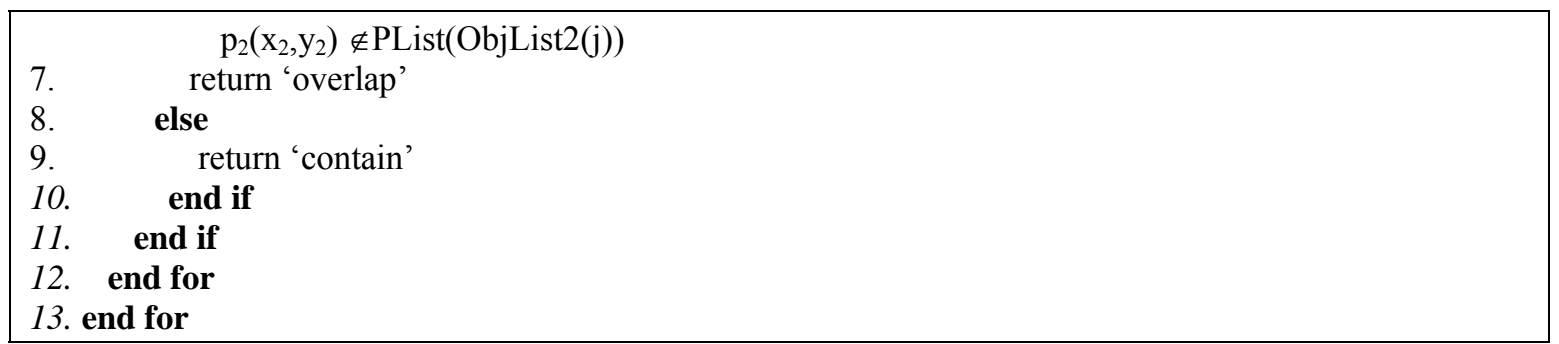

Figure 5. Algorithms for 3D spatial analysis

\subsection{FSM-based modeling}

In order to capture the evolution of object properties and inter-object spatial relationships over time, we use an event detection finite state machine (EDFSM). In this model, spatiotemporal event knowledge is represented in the form of different states and transitions among them. The states define different sub-events of a composite event, and the transitions represent the conditions required to move from one state to another. The spatio-temporal knowledge represented in EDFSM is used by the FSM transition function to identify occurrences of events in image sets. The transition function takes EDFSM representations of events of interest and finds any instances of those events in image sets. Extracted knowledge about such occurrences is stored in the form of an event representation graph (ERG). The ERG stores information about different states of the event occurrence, participating objects along with their spatial relations, and the time spent in each state.

\subsubsection{EDFSM}

Biological events involve complex interactions among objects and also evolution of objects over time. In order to capture the inter-object spatial relationships as well as the changes in the attributes of objects with the passage of time, we introduce the concept of an EDFSM. Formally, an EDFSM is a seven-tuple, 
$\left(\Sigma, \mathrm{T}, \Gamma_{\mathrm{O}}, \Gamma_{\mathrm{a}}, \Gamma_{\mathrm{s}}, \mathrm{I}, \Phi\right)$ where,

$\Sigma$ is a set of states, $\mathrm{T}$ is a set of transitions, $\Gamma_{\mathrm{o}}$ is a set of types of objects that make up an event, $\Gamma_{\mathrm{a}}$ is a set of conditions on the attributes of objects that make up an event, $\Gamma_{\mathrm{s}}$ is a set of conditions on the spatial projections of objects that make up the event, I is the set of initial states, and $\Phi$ is a set of final states. EDFSM can be represented graphically or using a state transition table. Figure 6 shows different components of an EDFSM.

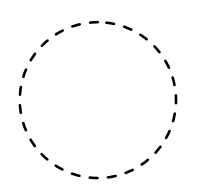

(a)

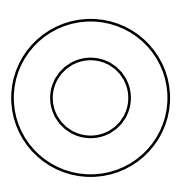

(b)

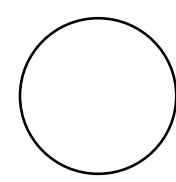

(c)

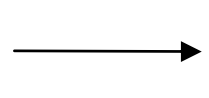

(d)

Figure 6. Symbols used for EDFSM and ERG. (a) start node (b) end node (c) intermediate node (d) transition.

\subsubsection{Event representation graph}

Detected events are represented using an ERG. Formally an $E R G$ is $G=(V, E)$ where $V$ is the set of vertices and $E$ is the set of edges. Each vertex is a three-tuple $(\mu, \eta, O)$ where $\mu$ is the start frame, $\eta$ is the duration, and $\mathrm{O}$ is the set of objects that constitute the event. Each edge represents a temporal relationship $\mathrm{T}$ between events where, $\mathrm{T} \in\{$ before, meets, overlaps, during, starts, equals, finishes $\}$

as defined in Table 1. Table 3 shows the functions used for maintenance of ERG.

\begin{tabular}{|l|l|}
\hline Function name & Explanation \\
\hline Create $(\mathrm{S})$ & Create node for state $\mathrm{S}$. \\
\hline Increment $(\mathrm{S})$ & Increment duration $\eta$ for the state $\mathrm{S}$. \\
\hline Rollback $\left(\mathrm{S}_{1}, \mathrm{~S}_{2}\right)$ & Rollback ERG from state $\mathrm{S}_{1}$ to $\mathrm{S}_{2}$. \\
\hline
\end{tabular}

Table 3. Functions for maintaining ERG. 


\subsubsection{FSM transition function}

Figure 7 describes the FSM transition function. The current state and the action are determined by the previous state and the input. State transition and action constraints are defined by means of the state transition table. FSM logic tries to identify if the event specified by EDFSM occurs in the time-lapse images. If any such event is identified, an ERG representation for that event is generated.

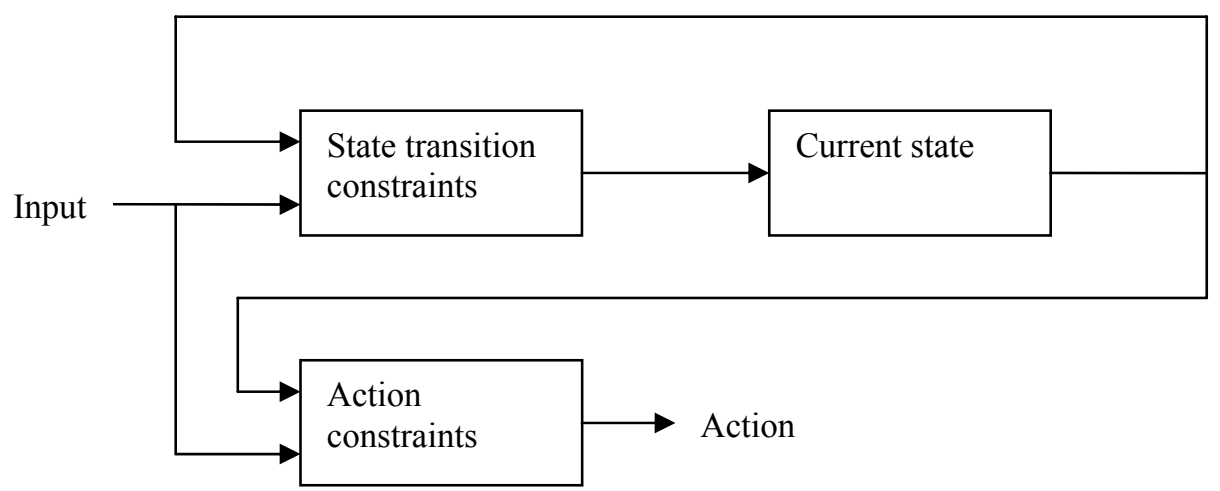

Figure 7. FSM transition function.

\subsection{Petri-net-based modeling}

FSM-based modeling works well for simple events. For more complicated events, for example multi-threaded events involving multiple objects with temporal constraints between constituent events, we use petri-nets [26]. A petri-net is a directed bipartitie graph which is used for modeling distributed systems. A petri-net contains place nodes, transition nodes, and directed arcs. Place nodes contain information about events and may be composed of simpler events. They may also contain delay nodes to express timing constraints between different events. This way a complex sequence of events can be identified. This composition of events can be used to 


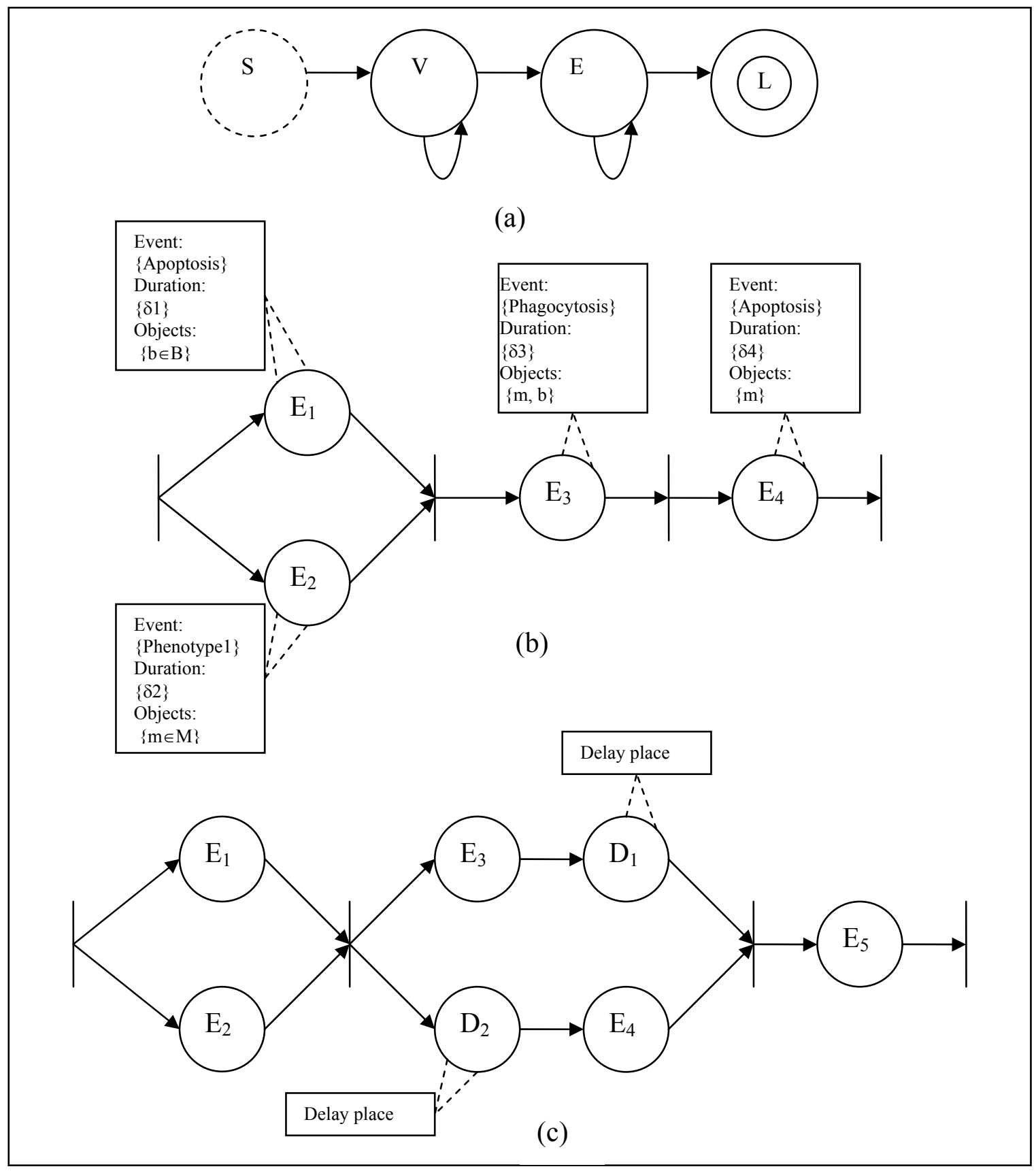

Figure 8. (a) EDFSM for apoptosis event. S, V, E, and L correspond to start, live, early apoptotic, and late apoptotic states, (b) A composite event represented using petri-net, (c) A composite event with delay places represented using petri-net. 


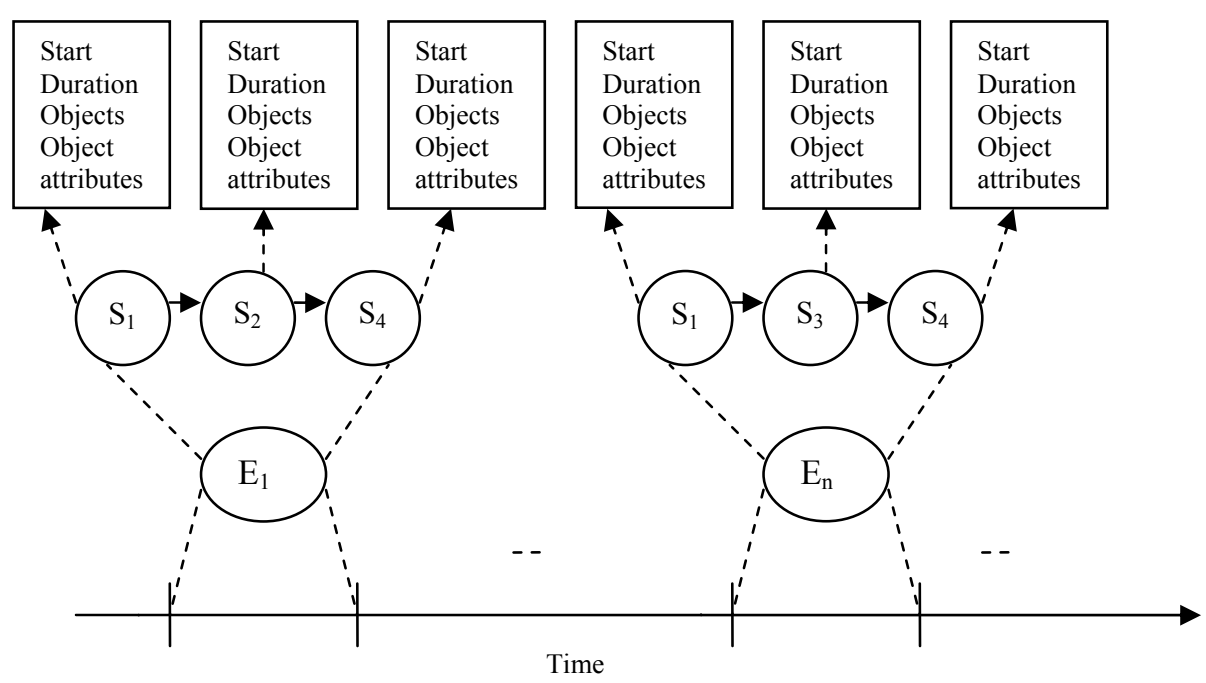

Figure 9. ERG representation of events in an imaging experiment

investigate the correlation between different events. In the example of Figure 8 (b), one may wish to query if phagocytosis of an apoptotic cell by a macrophage is followed by apoptosis of the macrophage. This can be achieved by finding all the instances in which the chain of events E1, E2, and E3 is followed by event E4 and then all the instances in which it is not. The probability that event E4 will happen after events E1, E2, and E3 can then be calculated. This mechanism can be used for discovering new knowledge from biological image sets. Figure 8 (c) shows an example event where timing constraints between sub-events of the composite event can also be specified using delay places.

\subsection{Semantic querying and event mining}

Different types of spatial and temporal events happen in a biological imaging study. Modeling and identification of these events can help answer important biological questions. The semantic event information extracted using the modeling techniques described above can be used to populate a database which makes conceptual queries and data mining possible. For instance, the effect of different drugs on different biological processes like apoptosis and phagocytosis can be studied using this approach. A graphical 
representation of different events happening over a period of time in an experiment is shown in Figure 9. A database populated with this information can be used to answer semantic queries like the following.

(a) What is the percentage increase in the number of phagocytosis events after the addition of a drug.

(b) How many cells follow a certain set of states undergoing apoptosis.

(c) Are two different types of spatio-temporal events correlated with each other.

Answering such queries in an objective and quantitative manner can significantly help in biological discovery.

\section{Examples}

In this section we demonstrate the expressive power of our model by encoding different events happening during apoptosis and phagocytosis.

\subsection{Time-lapse apoptosis screening example}

Apoptosis is defined as programmed cell death [27]. Study of apoptosis is important for cancer cell research. Many drugs are aimed at inducing apoptosis in cancer cells. Apoptosis can be detected by using a specific surface marker, for example Annexin V fluorescein isothiocyanate (FITC) and other markers for staining the nucleus and cytoplasm. Another stain called propidium iodide (PI) is used to verify cell viability. This marker cannot penetrate the cell membrane of living cells so it is not found in the nuclei of living cells. As the cell undergoes apoptosis, its cell membrane becomes more permeable and the dye stains the nucleus of the cell as well. A set of images depicting cells in different states is shown in Figure 10. Table 4 shows different states of cells during apoptosis along with their spatial constraints. Figure 8 (a) shows the EDFSM 
encoding to identify all live cells that go through early and late apoptotic states. The corresponding state transition table is shown in Table 5.

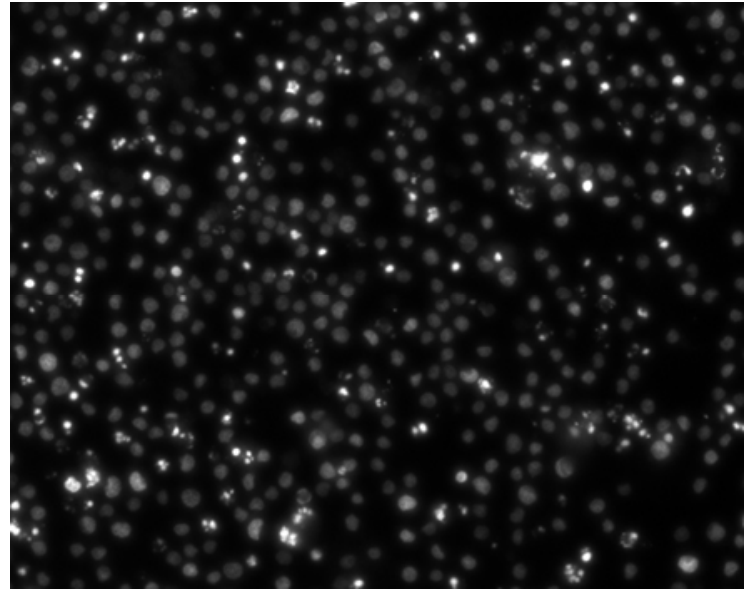

(a)

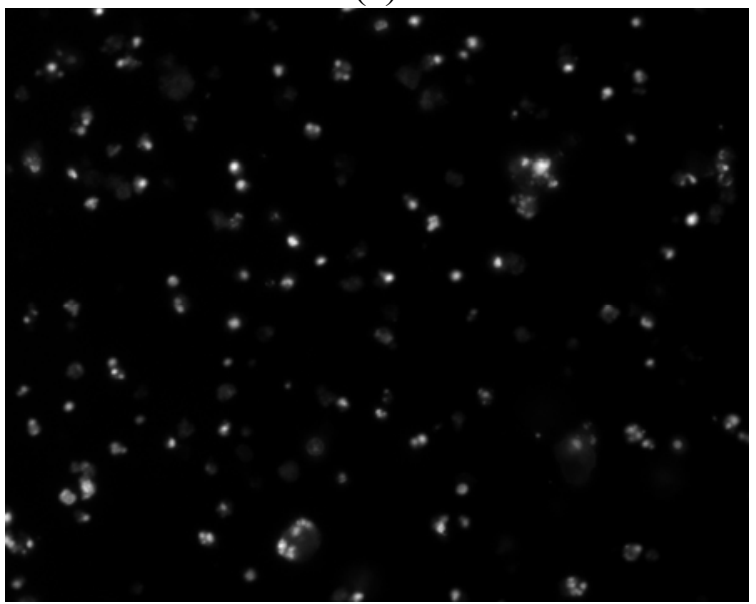

(c)

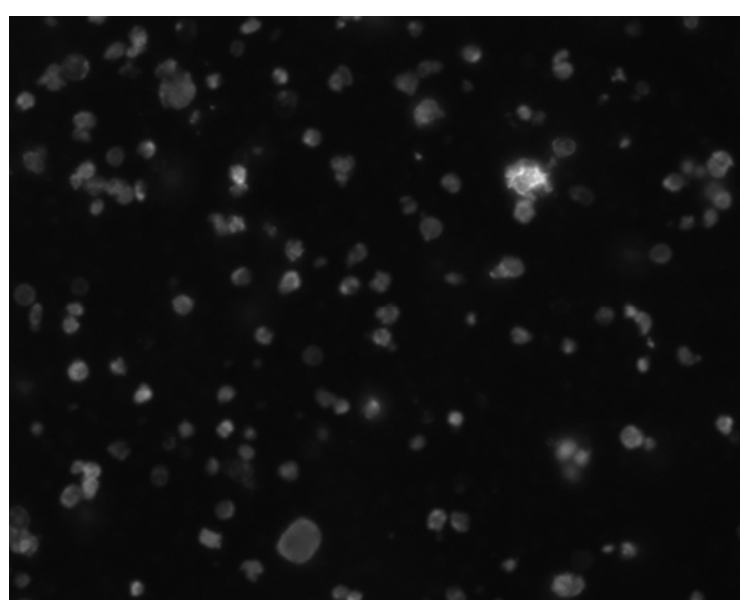

(b)

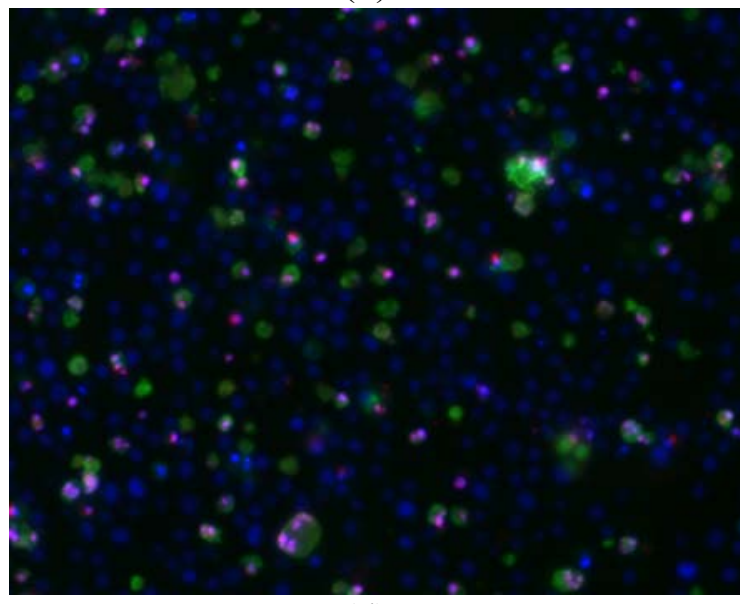

(d)

Figure 10. HL60 cells in different states during an apoptosis screen. (a) Hoechst 33342, (b) Annexin V FITC, (c) PI, (d) Merged image. The three images were pseudo-colored and merged to make the composite image. Blue, green and red colors correspond to Hoechst 33342, Annexin V FITC, and PI respectively. Cells in blue only are live, cells in blue and green are in early apoptotic state, and cells in blue, green, and red are in late apoptotic state.

\begin{tabular}{|l|l|}
\hline State & Spatial relations \\
\hline Live & Disjoint(H,A) \& Disjoint(H,P) \\
\hline Early apoptotic(EA) & Overlap(H, A) \& Disjoint(H,P) \\
\hline Late apoptotic(LA) & Overlap(H,A) \& Overlap(H,P) \\
\hline
\end{tabular}

Table 4. Cell states and corresponding spatial relations between Hoechst $(H)$, Annexin-V$\operatorname{FITC}(\mathrm{A})$, and $\mathrm{PI}(\mathrm{P})$. 


\begin{tabular}{|l|l|l|l|l|}
\hline Current state & Objects & $\begin{array}{l}\text { Spatial } \\
\text { Relations }\end{array}$ & Next state & Action \\
\hline S & H & Live & V & Cr(S1) \\
\hline V & H & Live & V & Inc(S1) \\
\hline V & H, A & EA & E & Cr(S2) \\
\hline E & H, A & EA & E & Inc(S2) \\
\hline E & H, A, P & LA & L & Cr(S3) \\
\hline
\end{tabular}

Table 5. State transition table corresponding to the EDFSM of Figure 5. Actions $\mathrm{cr}$ and inc correspond to the create and increment functions that create a node in ERG or increment the duration of a node in ERG. Spatial relations refer to the ones defined in Table 2.
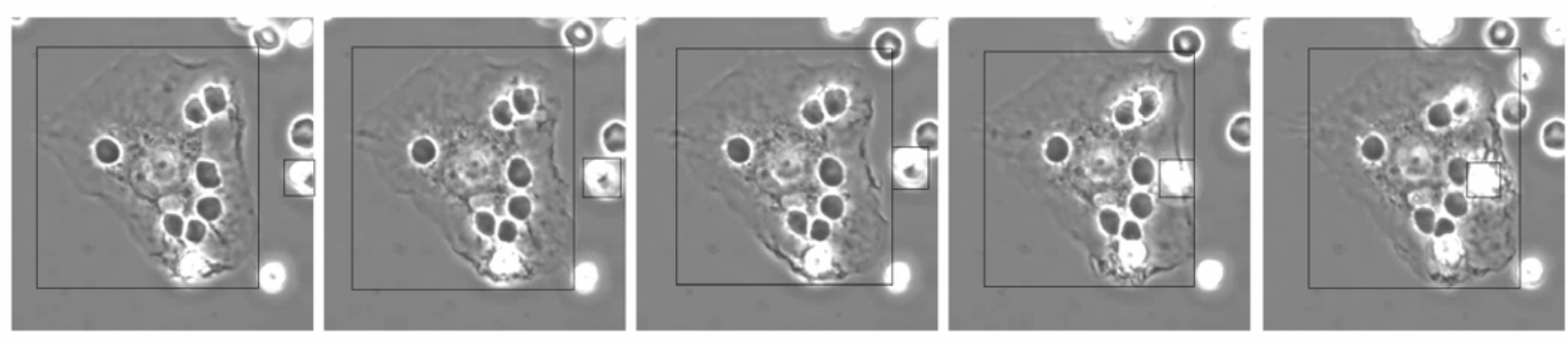

Figure 11. Sequence of events constituting a phagocytosis event

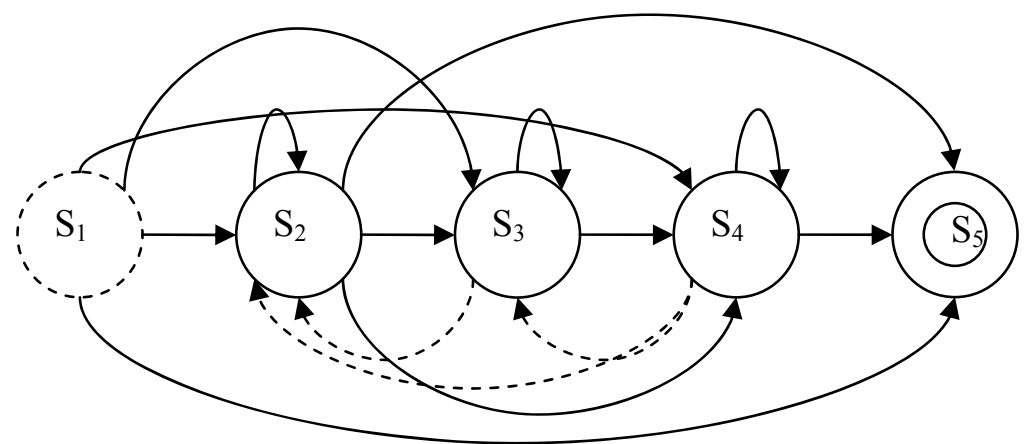

Figure 12. EDFSM for a phagocytosis event, S1, S2, S3, S4, S5 correpond to start, disjoint, ingestion_start, ingestion_complete and phagocytosis_complete.

\subsection{Phagocytosis}

We next give an example of a multiple-object event. Phagocytosis is defined as the process of engulfment of a microorganism or an object by cells. This process is essential for body defense against harmful bacteria and infections. We consider the sequence of 
spatial events that make up the composite event "phagocytosis" as shown in Figure 11 [28]. We next present FSM representation of the sequence of events. The process involves the detection, engulfment, and ingestion of microorganisms. Sometime it is also followed by an oxidative burst. The process can be detected by following the movements of different objects. The sequence of events in Figure 11 shows the phagocytosis of erythrocytes by macrophages. As is obvious, the erythrocyte and the macrophage are initially at disjoint positions. At a later time the distance between the two decreases and they have an overlap for some time, after which the erythrocyte is ingested. Figure 12 shows the FSM representation of this composite event and Table 6 shows the corresponding state transition table.

\begin{tabular}{|l|l|c|l|l|l|}
\hline $\begin{array}{l}\text { Current } \\
\text { state }\end{array}$ & $\begin{array}{l}\text { Object } \\
\text { constraints }\end{array}$ & $\begin{array}{l}\text { Object attribute } \\
\text { constraints }\end{array}$ & $\begin{array}{l}\text { Spatial } \\
\text { constraints }\end{array}$ & $\begin{array}{l}\text { Next } \\
\text { state }\end{array}$ & Action \\
\hline$S_{1}$ & $m \in M, e \in E$ & - & Disjoint $(m, e)$ & $S_{2}$ & Create $\left(S_{2}\right)$ \\
\hline$S_{1}$ & $m \in M, e \in E$ & - & Touch $(m, e)$ & $S_{3}$ & Create $\left(S_{3}\right)$ \\
\hline$S_{1}$ & $m \in M, e \in E$ & - & Overlap $(m, e)$ & $S_{4}$ & Create $\left(S_{4}\right)$ \\
\hline$S_{1}$ & $m \in M, e \in E$ & Brightness $(e)<$ threshold & Enclosed $(m, e)$ & $S_{5}$ & Create $\left(S_{5}\right)$ \\
\hline$S_{2}$ & $m \in M, e \in E$ & - & Disjoint $(m, e)$ & $S_{2}$ & Inc $\left(S_{2}\right)$ \\
\hline$S_{2}$ & $m \in M, e \in E$ & - & Touch $(m, e)$ & $S_{3}$ & Create $\left(S_{3}\right)$ \\
\hline$S_{2}$ & $m \in M, e \in E$ & - & Overlap $(m, e)$ & $S_{4}$ & Create $\left(S_{4}\right)$ \\
\hline$S_{2}$ & $m \in M, e \in E$ & Brightness $(e)<$ threshold & Enclosed $(m, e)$ & $S_{5}$ & Create $\left(S_{5}\right)$ \\
\hline$S_{3}$ & $m \in M, e \in E$ & - & Touch $(m, e)$ & $S_{3}$ & Inc $\left(S_{3}\right)$ \\
\hline$S_{3}$ & $m \in M, e \in E$ & - & Overlap $(m, e)$ & $S_{4}$ & Create $\left(S_{4}\right)$ \\
\hline$S_{3}$ & $m \in M, e \in E$ & Brightness $(e)<$ threshold & Enclosed $(m, e)$ & $S_{5}$ & Create $\left(S_{5}\right)$ \\
\hline$S_{3}$ & $m \in M, e \in E$ & - & Disjoint $(m, e)$ & $S_{2}$ & RB $\left(S_{3}, S_{2}\right)$ \\
\hline$S_{4}$ & $m \in M, e \in E$ & - & Overlap $(m, e)$ & $S_{4}$ & Inc $\left(S_{4}\right)$ \\
\hline$S_{4}$ & $m \in M, e \in E$ & Brightness $(e)<$ threshold & Enclosed $(m, e)$ & $S_{5}$ & Create $\left(S_{5}\right)$ \\
\hline$S_{4}$ & $m \in M, e \in E$ & - & Touch $(m, e)$ & $S_{3}$ & RB $\left(S_{4}, S_{3}\right)$ \\
\hline$S_{4}$ & $m \in M, e \in E$ & - & Disjoint $(m, e)$ & $S_{2}$ & $\operatorname{RB}\left(S_{4}, S_{2}\right)$ \\
\hline
\end{tabular}

Table 6. State transition table corresponding to the EDFSM of Figure 10. Actions create, inc and rb create a node in ERG, increment duration, and rollback to an appropriate state respectively. 


\section{Experimental results and discussion}

\subsection{Spatial analysis}

In this section, we present the results of a comparison between the algorithms presented in section 3.1. Relationship accuracy and speed are our performance criteria. We used images generated by a particle delivery study [29]. Delivery of gold particles to subcellular locations can enhance the Raman signal of the molecules that are located in close proximity and hence increases the detection sensitivity. In order to quantitatively investigate the localization of gold particles inside cells, cells were stained with nucleus and cytoplasm specific dyes and were subsequently imaged using confocal microscopy. Nuclei and gold particles were segmented using a manually defined threshold, and cytoplasm was segmented using the algorithm described in [30]. This generated three images for each set. A representative set is shown in Figure 13. For our experiments we used 13 sets of images containing 119 cells and 2149 gold particles. Spatial relationship of gold particles was established with nuclei and cytoplasm. As it is not feasible to manually validate the accuracy for this large number of spatial comparisons $(\sim 20,000)$, we use EO algorithm as the baseline. EO algorithm provides the most accurate spatial representation. The only inaccuracies that it may have are the result of segmentation errors, which are not the focus of this paper. We check whether the gold particles are disjoint, have a partial overlap or are contained within the nuclei or cytoplasm. The accuracy is determined in terms of the number of gold particles that are correctly assigned to the objects that they are actually contained in or have a partial overlap with. Table 7 presents the comparison of accuracy for different algorithms. Next, we present the time taken by each of these algorithms in Table 8 . As can be seen the accuracy of BB as well as $\mathrm{CH}$ is higher for nucleus than for cytoplasm. This is so because both the $\mathrm{BB}$ 
and $\mathrm{CH}$ are a better spatial representation for nucleus than for cytoplasm as is shown in Figure 14. For the same reason hybrid3 algorithm gives a speedup of $\sim 14$ for nuclei and only $\sim 4$ for cytoplasm (EO algorithm is used as baseline for speedup). This implies that the choice of algorithm for a particular application depends on the shape of the objects. Also, BB algorithm is the fastest but the least accurate whereas EO and hybrid algorithms using EO are the most accurate. From the results, it is clear that hybrid algorithms using $\mathrm{BB}$ and $\mathrm{EO}$ or $\mathrm{BB}, \mathrm{CH}$, and $\mathrm{EO}$ are to be preferred when maximum accuracy is needed. Inter-object spatial relations can be used for studying localization of objects and different aspects of the distribution of particles within cells in a quantitative manner. Figure 15 shows histograms of the area of particles that are distributed in cytoplasm and nuclei.

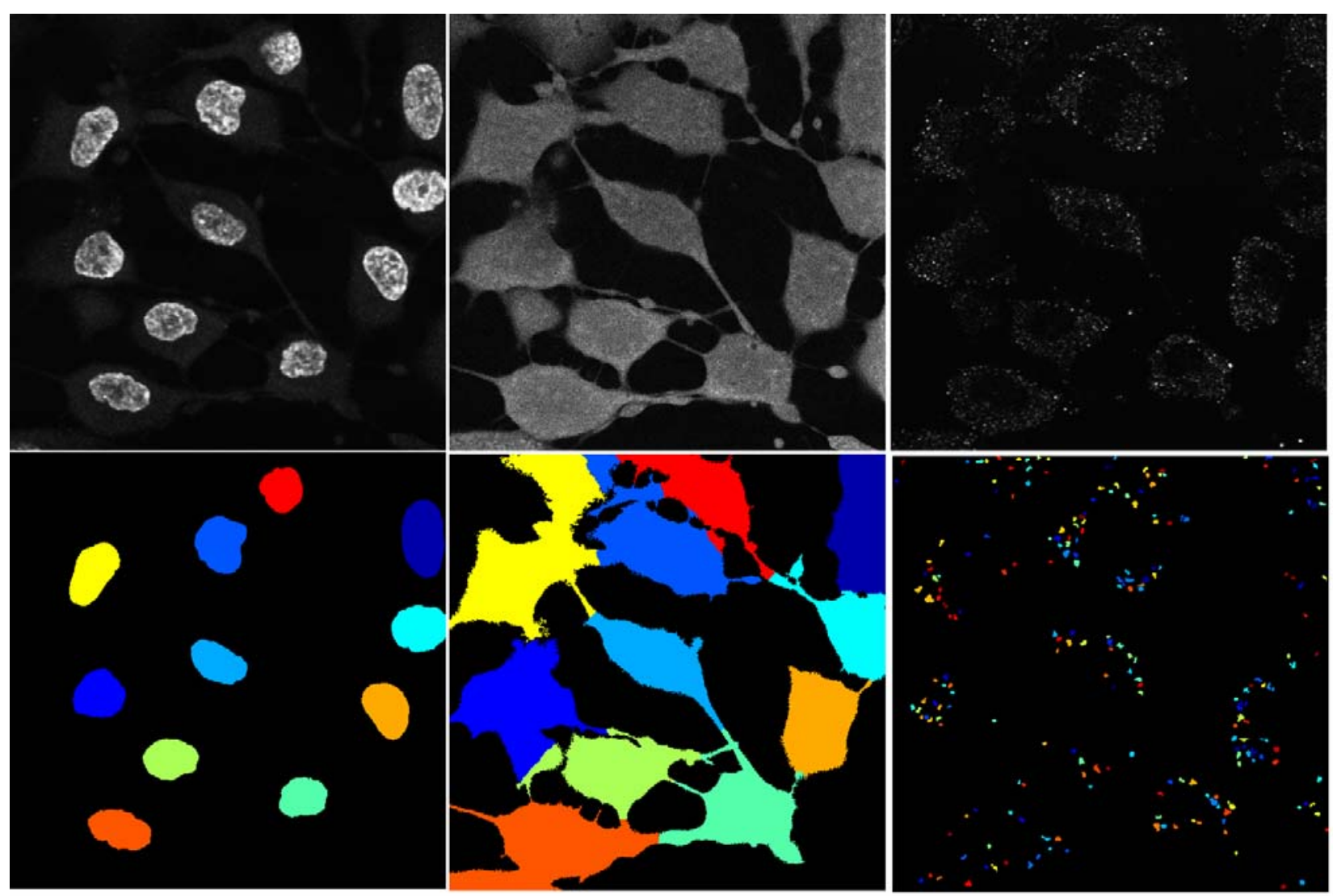

Figure 13. A representative set of images, (Upper left) Nuclei, (Lower left) Segmented nuclei, (Upper middle) Cytoplasm, (Lower middle) Segmented cytoplasm, (Upper right) Gold particles, (Lower right) Segmented gold particles. 


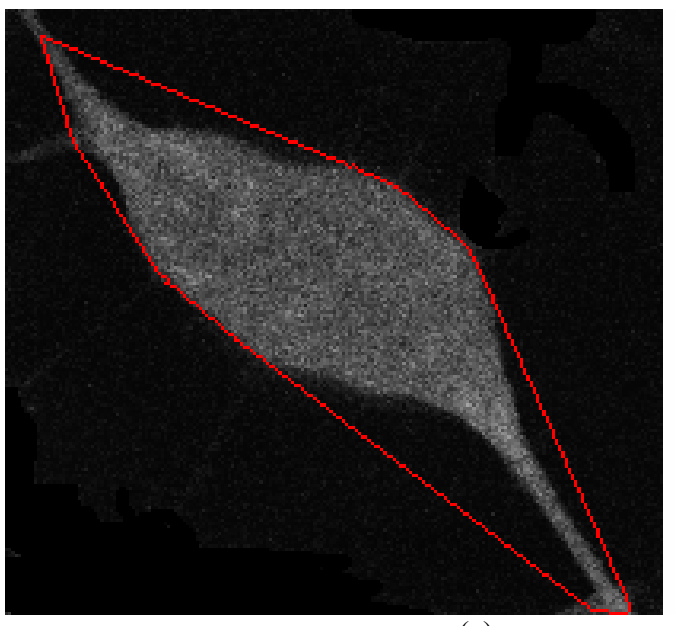

(a)

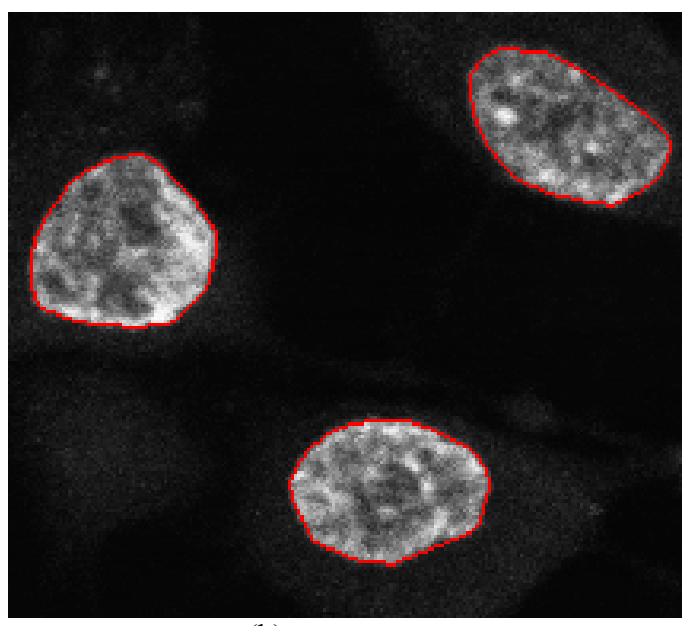

(b)

Figure 14. (a) Convex hull of cytoplasm, (b) Convex hull of nuclei. Convex hull is a better spatial representation for nuclei than cytoplasm.

\begin{tabular}{|l|l|l|}
\hline Algorithm & $\begin{array}{l}\text { Accuracy for } \\
\text { nuclei (\%) }\end{array}$ & $\begin{array}{l}\text { Accuracy for } \\
\text { cytoplasm (\%) }\end{array}$ \\
\hline Bounding box (BB) & 82.41 & 71.75 \\
\hline Convex hull $(\mathrm{CH})$ & 96.51 & 87.25 \\
\hline Exact outline $(\mathrm{EO})$ & 100 & 100 \\
\hline Hybrid1 $(\mathrm{BB}+\mathrm{CH})$ & 96.51 & 87.25 \\
\hline Hybrid2 $(\mathrm{BB}+\mathrm{EO})$ & 100 & 100 \\
\hline Hybrid3(BB+CH+EO) & 100 & 100 \\
\hline
\end{tabular}

Table 7. Comparison of accuracy of different algorithms. EO algorithm is used as the base case.

\begin{tabular}{|l|l|l|}
\hline Algorithm & Time for nuclei (sec) & Time for cytoplasm (sec) \\
\hline Bounding box $(\mathrm{BB})$ & 6.766 & 6.7809 \\
\hline Convex hull $(\mathrm{CH})$ & 101.17 & 111.53 \\
\hline Exact outline $(\mathrm{EO})$ & 317.48 & 1385 \\
\hline Hybrid1 $(\mathrm{BB}+\mathrm{CH})$ & 13.24 & 31.62 \\
\hline Hybrid2 $(\mathrm{BB}+\mathrm{EO})$ & 25.59 & 441.48 \\
\hline Hybrid3 (BB+CH+EO) & 22.88 & 354.77 \\
\hline
\end{tabular}

Table 8. Comparison of speed of different algorithms. 


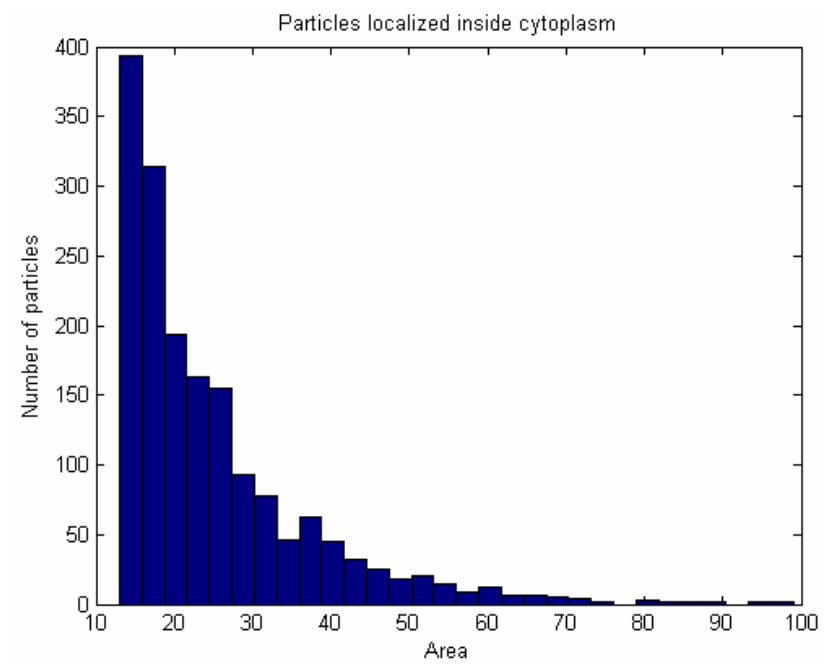

(a)

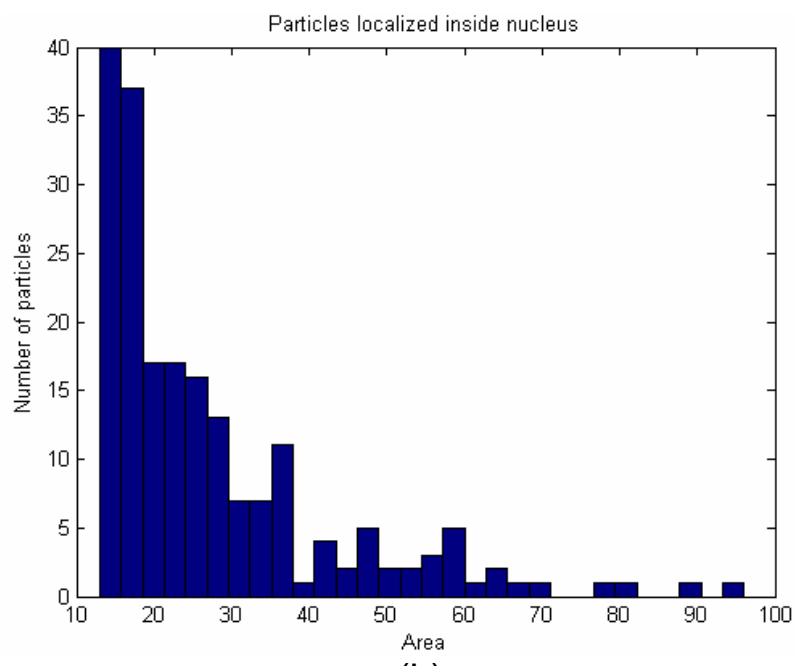

(b)

Figure 15. Particles localized inside cells (a) Histogram of the area of particles localized inside cytoplasm. (b) Histogram of the area of particles localized inside nucleus.

In order to evaluate the 3D spatial-analysis algorithms, we used simulated 3D confocal data. Two data sets were generated: a smaller set containing 20 confocal slices with each slice having a size of $100 \times 100$ pixles, and a larger set containing 50 confocal slices with each slice having a size of 500x500 pixels. The results of the 3D spatial analysis are shown in Table 9 for the two data sets. Pixel comparison-based analysis is the fastest. Although the bounding box-based spatial analysis itself is very fast, the computation of bounding boxes for a large number of objects in 3D is time consuming and responsible for the overall slow speed of this method. Pixel-level comparison also has the best accuracy of the three algorithms.

\begin{tabular}{|l|l|l|}
\hline Algorithm & Large $(\mathrm{sec})$ & Small $(\mathrm{sec})$ \\
\hline 3DBB & 10.1107 & 0.5500 \\
\hline 3DHybrid & 4.8636 & 0.1852 \\
\hline 3DPixComp & 3.6597 & 0.0664 \\
\hline
\end{tabular}

Table 9. Comparison of the runtime for different 3D-spatial-analysis algorithms. An image set containing 50 z-slices was used. Each slice was $500 \times 500$ pixels. 


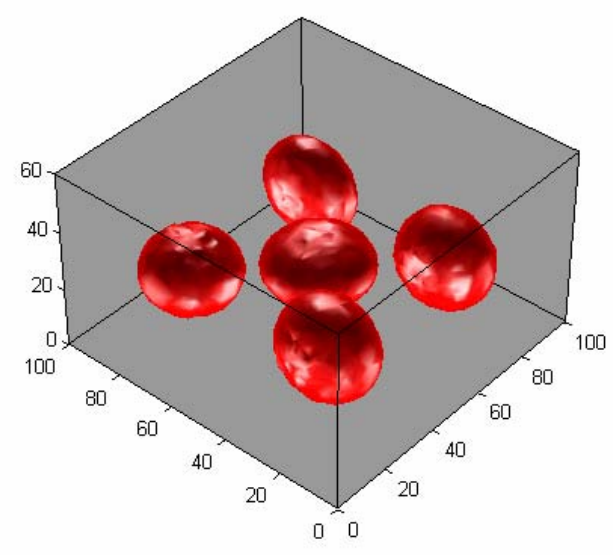

(a)

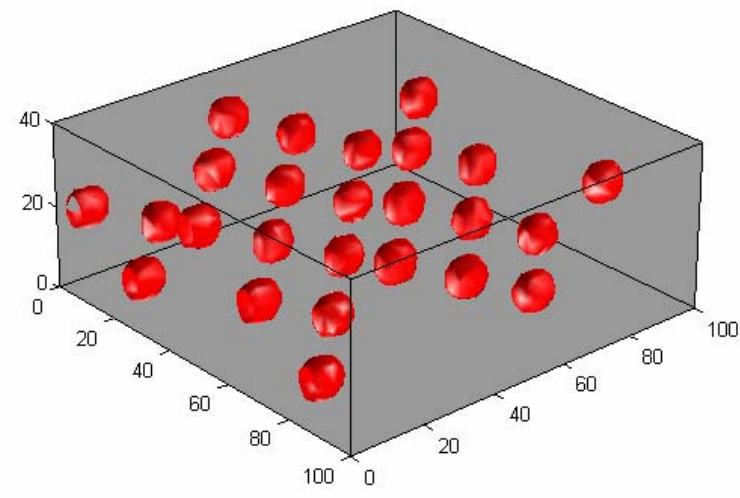

(b)

Figure 16. (a) Surface rendering of the 3D confocal data set simulating cells (b) Surface rendering of the 3D confocal data set simulating particles.

\subsection{Temporal analysis}

The extra information provided by per-cell analysis can be used to compensate for experimental discrepancies. Human cervical cancer cells (HeLa) were treated with an apoptosis-inducing drug (camptothecin) and were imaged using an imaging cytometer (iCys research imaging cytometer, CompuCyte Corporation, Cambridge, MA) every thirty minutes for 4 and a half hours. Image registration issues were resolved, and cells were segmented and tracked. The numbers of live, early apoptotic, and late apoptotic cells were found for every frame of image sequence on a population basis using the spatial relations defined in Table 4. The results are shown in Figure 17 (a). The cells were then analyzed on a per-cell basis. Time profile of two different cells is shown below.

\section{V E E L L E L L}

\section{V V E E L L V L}

Cells can only transition from live to early apoptotic and from early apoptotic to late apoptotic state. Hence the transition from $\mathrm{L} \rightarrow \mathrm{E}$ and $\mathrm{L} \rightarrow \mathrm{V}$ is not possible. Sources of 
error may include photobleaching of dyes, inconsistent illumination, and faulty autofocus module. This inaccuracy can however be fixed if single cells are tracked during the experiment. Rules can be defined that check for inconsistent state transitions and remove the discrepancy. The revised results are shown in Figure 17 (b). These results were obtained by checking the previous state for each current state and removing all inconsistent state transitions. We would like to mention that a careful study of the sources of error is required for choosing appropriate rules for artifact removal. Spatio-temporal modeling is useful not only for removing artifacts from experimental data but also for studying the behavior of large populations of cells on a per-cell basis.

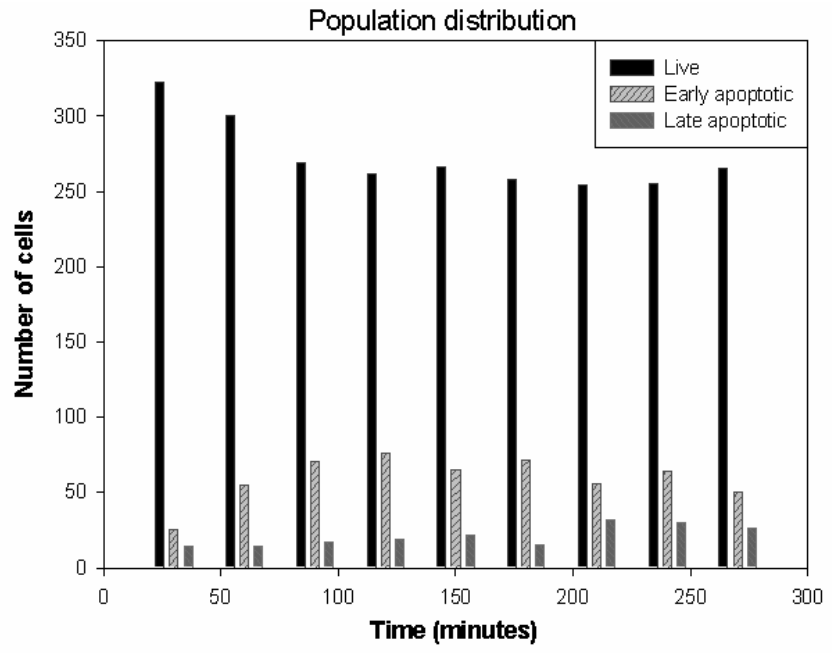

(a)

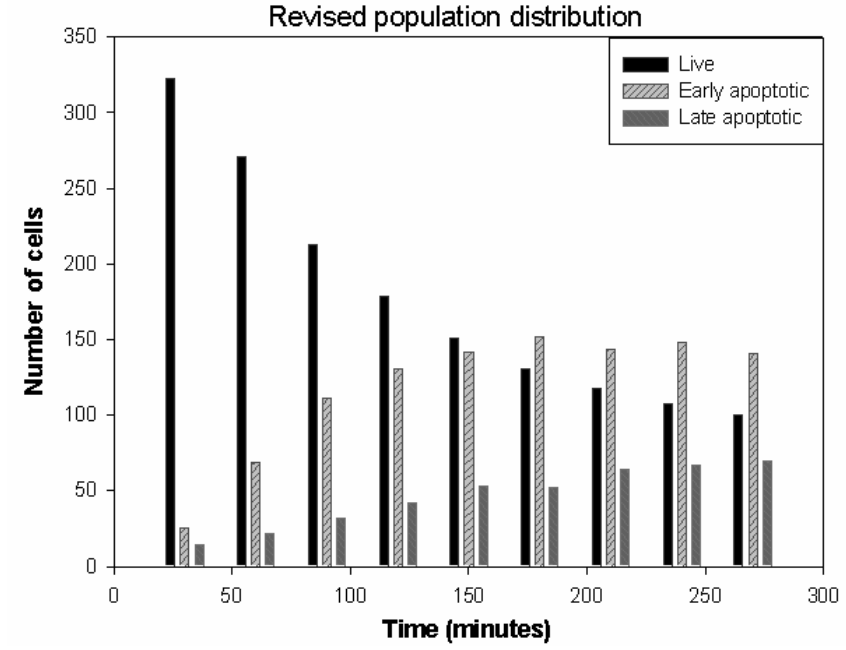

(b)

Figure 17. (a) Original population counts for time-lapse apoptosis study (b) Revised population counts for time-lapse apoptosis study

\subsection{Validation}

As HCS technologies generate large volume of data, it is not feasible to manually validate analysis algorithms [31]. In our case, the data set contains 363 cells. This means 
visual validation will require inspecting 363 × 9 × $3=9801$ objects, which is time consuming and prone to human error. Validation, therefore, requires modeling the data generation process. We model the data generation process for the apoptosis screen as a Markov chain with state space $\mathrm{S}$, initial distribution $\Pi$ and transition matrix $\mathrm{P}$. As most cells in the beginning are in live state, it is given a high probability in $\Pi$. We also assume that the probability of a cell moving to the immediate next state is equal to it maintaining its current state while it is in state $\mathrm{V}$ or $\mathrm{E}$. In state $\mathrm{V}$, cells are more likely to move to state E than moving directly to state $\mathrm{L}$ hence the transition $\mathrm{V} \rightarrow \mathrm{L}$ is given half the probability of the transition $\mathrm{V} \rightarrow \mathrm{E}$. The state $\mathrm{L}$ is assumed to be an absorbing state..

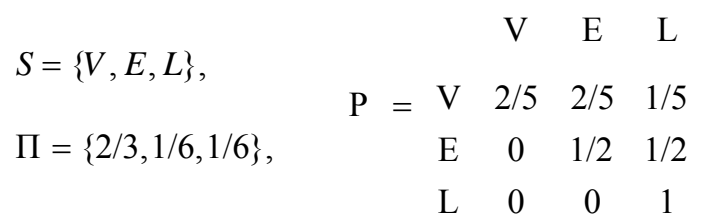

Next we model system error as a process that changes a particular state to an earlier state with probability $\mathrm{p}$, which is the parameter of our system. With this model for data generation we report the performance of our error correction rule in Table 10 for 1000 cells imaged at 9 time points. As can be seen, a significant number of artifacts is removed.

\begin{tabular}{|l|l|l|l|}
\hline$p$ & No. of artifacts & $\begin{array}{l}\text { No. of artifacts } \\
\text { removed }\end{array}$ & \% artifacts removed \\
\hline $1 / 10$ & 793 & 616 & 77.68 \\
\hline $1 / 5$ & 1656 & 1238 & 75.85 \\
\hline $1 / 3$ & 2643 & 1949 & 73.74 \\
\hline $1 / 2$ & 3876 & 2590 & 66.82 \\
\hline $2 / 3$ & 5153 & 2852 & 55.35 \\
\hline
\end{tabular}

Table 10. Performance of artifact removal rule on synthetic data. 


\section{Conclusion and future work}

Analysis of massive amount of multimedia biological data generated by HCS technologies requires intelligent multimedia analysis and knowledge extraction tools. Integration of phenotypic information with genomic and proteomic information requires modeling at multiple levels. It is important to extract not only the low-level image features but also the high-level semantic information in terms of biological events. Quantitative analysis of this high-level spatio-temporal information in response to different environmental and experimental conditions provides insights into the working of biological cells and organisms. In this work we have demonstrated how high-level semantic information can be extracted using different spatio-temporal models. In the future we plan to apply these tools to more biological applications and explore different algorithms for event mining for discovering biological knowledge.

\section{References}

1. K. A. Giuliano, "High-content screening: A new approach to easing key bottlenecks in the drug discovery process", Journal of Biomolecular Screening, Vol. 2, No. 4, 1997, pp. 249-259.

2. Xiaobo Zhou, S. T. C Wong, "Informatics challenges of high-throughput microscopy", IEEE Signal Processing Magazine, Vol. 23, Issue 3, May 2006, pp. 63-72.

3. J. Chen et al., "Grand challenges for multimodal bio-medical systems", IEEE Circuits and Systems Magazine, Vol. 5, Issue 2, 2005, pp. 46-52.

4. J. C. Venter et al., "The sequence of the human genome”, Science, Vol. 291, No. 5507, 16 February 2001, pp. 1304-1351. 
5. D. J. Stephens, V. J. Allan, "Light microscopy techniques for live cell imaging", Science, 4 April 2003, pp. 82-86.

6. M. E. Dickinson, G. Bearman, S. Tille, R. Lansford, S. E. Fraser, "Multi-spectral imaging and linear unmixing add a whole new dimension to laser scanning fluorescence microscopy,” Biotechniques, Vol. 31, No. 6, 2001, pp. 1272-1278.

7. N. Dimitrova, "Context and memory in multimedia content analysis", IEEE Multimedia, Vol. 11, Issue 3, Jul-Sept. 2004, pp. 7-11.

8. F. Jianping, L. Hangzai, A. K. Elmagarmid, "Concept-oriented Indexing of Video Databases: Toward Semantic Sensitive Retrieval and Browsing," IEEE Transactions on Image Processing, Vol. 13, No.7, July 2004.

9. W. W. Chu, C. Hsu, A. F. Cardenas, R. K. Taira, "Knowledge-Based Image Retrieval with Spatial and Temporal Constructs", IEEE Transactions on Knowledge and Data Engineering, Vol. 10, No. 6, November/December 1998.

10. C. Zimmer et al., "On the digital trail of mobile cells," IEEE Signal Processing Magazine, Vol. 23, Issue 3, May 2006, pp. 54-62.

11. T. R. Jones, A. E Carpenter, P. Golland and D. M. Sabatini, Methods for highcontent, high-throughput image-based cell screening, in Proc. of the Workshop on Microscopic Image Analysis with Applications in Biology (MIAAB), Copenhagen, Denmark, 2006, pp 65-72.

12. W. M. Ahmed, M. N. Ayyaz, B. Rajwa, F. Khan, A. Ghafoor, J. P. Robinson, "Semantic analysis of biological imaging data: challenges and opportunities," International Journal of Semantic Computing, Vol. 1, No. 1, 2007, pp. 67-85. 
13. P. Bamford, "Empirical comparison of cell segmentation algorithms using an annotated dataset," in Proc. International Conference on Image Processing, 2003, pp. II1073-1076.

14. A. Garrido, N. Perez de la Blanca, Applying deformable templates for cell image segmentation, Pattern Recognition, 33, 2000, pp. 821-832.

15. M. Hu, X. Ping and Y. Ding, Automated cell nucleus segmentation using improved snake, in Proc. International Conference on Image Processing, 2004, pp. 2737-2740.

16. T. R. Jones, A. E. Carpenter, P. Golland, "Voronoi-based segmentation of cell on image manifolds", Proceedings of the ICCV Workshop on Computer Vision for Biomedical Image Applications, Beijing, China, October 21, Lecture Notes in Computer Science 3765, Springer-Verlag, Berlin, pp. 535-543.

17. A. K. Jain, R. P. W. Duin, J. Mao, "Statistical pattern recognition: a review", IEEE Transactions on Pattern Analysis and Machine Intelligence, Vol. 22, No. 1, January 2000, pp. 4-37.

18. E. Meijering, I. Smal and G. Danuser, Tracking in molecular bioimaging, IEEE Signal Processing Magazine, Vol. 23, Issue 3, May 2006, pp. 46-53.

19. M. K. Cheezum, W. F. Walker and W. H. Guilford, "Quantitative comparison of algorithms for tracking single fluorescent particles," Biophysical Journal, Vol. 81, No. 4, 2001, pp. 2378-2388.

20. C. Zimmer, E. Labruyere, V. Meas-Yedid, N. Guillen and J. Olivo-Marin, "Segmentation and tracking of migrating cells in video microscopy with parametric active contours: A tool for cell-based drug testing," IEEE Trans. on Medical Imaging, Vol. 21, No. 10, October 2002. 
21. W. M. Ahmed et al., “An XML-based system for providing knowledge-based grid services for high-throughput biological imaging”, IEEE Transactions on Information Technology in Biomedicine (submitted).

22. J. F. Allen, "Maintaining knowledge about temporal intervals", Communications of the ACM, November 1983, pp. 832-843.

23. Y. F. Day, S. Dagtas, M. Iino, A. Khokhar, A. Ghafoor, “Object-oriented conceptual modeling of video data”, 11th International Conference on Data Engineering(ICDE'95), 1995, pp. 401-408.

24. M. Vazirgiannis, Y. Theodoridis, T. Sellis, "Spatio-temporal composition and indexing for large multimedia applications", Multimedia Systems, Vol. 6, No. 4, July 1998.

25. J. O'Rourke, “Computational geometry in C”, Cambridge University Press, New York, 2005.

26. W. Al-Khatib, Arif Ghafoor, "An approach for video meta-data modeling and query processing", Proceeding of the Seventh ACM International Conference on Multimedia (Part 1), 1999, pp. 215-224.

27. J. D. Robertson, S. Orrenius, B. Zhivotovsky, "Review: nuclear events in apoptosis", Journal of Structural Biology, Volume 129, Issues 2-3, April 2000, pp. 346-358.

28. Diakonova, M. et al., "Dynamics of Cytoskeletal Proteins during Fc $\gamma$ Receptormediated Phagocytosis in Macrophages", Molecular Biology of the Cell, Vol. 13, 402411, February 2002. Images reprinted with the permission of The American Society for Cell Biology. 
29. A. Shamsaie, M. Jonczyk, J. Sturgis, J. P. Robinson, J. Irudayaraj, "Intracellularly grown gold nanoparticles (IGAuN) as potential SERS probes", Journal of Biomedical Optics, (in press).

30. T. R. Jones, A. E. Carpenter, P. Golland, "Voronoi-based segmentation of cells on image manifolds", Proceedings of the ICCV Workshop on Computer Vision for Biomedical Image Applications (CVBIA). Yanxi Liu, Tianzi Jiang, Changshui Zhang (Eds.). Beijing, China, October 21. Lecture Notes in Computer Science 3765. Published by Springer-Verlag, Berlin, p. 535-543, ISBN 3-540-29411-2.

31. X. Zhou, S. T. C. Wong, "Informatics challenges of high-throughput microscopy", IEEE Signal Processing Magazine, May 2006, pp. 63-72. 